\title{
1 Organs-on-chips: into the next decade
}

2 Lucie A. Low $\mathrm{PhD}^{1+}$; Christine Mummery $\mathrm{PhD}^{2,3}$; Brian R. Berridge ${ }^{4}$; Christopher P. Austin MD ${ }^{1}$;

3 Danilo A. Tagle $\mathrm{PhD}^{1+}$

$4 \quad{ }^{1}$ National Center for Advancing Translational Sciences, National Institutes of Health, USA

$5{ }^{2}$ Leiden University Medical Center, the Netherlands

$6{ }^{3}$ University of Twente, the Netherlands

$7 \quad{ }^{4}$ National Institute for Environmental Health Sciences, National Institutes of Health, USA

$8{ }^{\dagger}$ Co-corresponding author emails: lucie.low@nih.gov and danilo.tagle@nih.gov 
11 Organs-on-chips (OoCs), also known as microphysiological systems or "tissue chips" (the terms

12 are synonymous), have garnered substantial interest in recent years owing to their potential to

13 be informative at multiple stages of the drug discovery and development process. These

14 innovative devices could provide insights into normal human organ function and disease

15 pathophysiology, as well as more accurately predict the safety and efficacy of investigational

16 drugs in humans. Therefore, they are likely to become useful additions to traditional preclinical

17 cell culture methods and in vivo animal studies in the near term, and in some cases,

18 replacements for them in the longer term. In the last decade, the OoC field has seen dramatic

19 advances in the sophistication of biology and engineering, in the demonstration of physiological

20 relevance, and in the range of applications. These advances have also revealed new challenges

21 and opportunities, and expertise from multiple biomedical and engineering fields will be

22 needed to fully realize the promise of OoCs for fundamental and translational applications. This

23 Review provides a snapshot of this fast-evolving technology, discusses current applications and

24 caveats for their implementation, and offers suggestions for directions in the next decade. 
27 Drug development is slow and costly, driven mainly by high attrition rates in clinical trials ${ }^{1}$. Although remarkable increases in our understanding of the molecular underpinnings of human diseases and our ability to model in vivo cell, tissue and organ-level biology have been made over the past three decades, the number of US Food and Drug Administration (FDA)-approved drugs per billion US\$ spent on research and development has actually decreased monotonically since $1950^{2}$. Drug development needs new approaches, paradigms and tools to reverse these trends and thus deliver on the promise of science for patients ${ }^{2}$.

Although animal models have contributed enormously both to our understanding of physiology and disease, and to the development of new medicines, researchers have long been aware of the frequent discordance between animal and human studies and therefore the need for modeling and testing platforms that would be more predictive of human responses ${ }^{3,4}$. Indeed, drug candidates may be terminated for lack of efficacy in animals, or discovery of hazards or toxicity in animals that might not be human-relevant. Despite significant developments in computational and in vitro biology and toxicology in the last two decades, currently over $80 \%$ of

42 investigational drugs fail in clinical testing, with $60 \%$ of those failures due to lack of efficacy and 43 another $30 \%$ due to toxicity ${ }^{5}$.

To address some of these issues and offer alternative tools for preclinical stages, early "cell culture analogs" ${ }^{\prime 67}$ were explicitly designed to culture mammalian cells in linked chambers

47 perfused with a recirculating tissue medium, or "blood surrogate". Following on from these 48 models came a "heart-lung micromachine", integrating a lung cell culture model with a cardiac 49 device to assess the effects of drugs and therapeutics delivered to the human lung by aerosol 50 on cardiac function and toxicity in vitro. This first "lung-on-a-chip" research was published in $512010^{8}$ and set the stage for organs-on-chips (OoCs, synonymously known as "tissue chips" or 52 microphysiological systems (MPS)) - microdevices engineered to contain (human) cells and 53 tissues and to model or mimic organ structures, functions, and reactions to biological 54 conditions, stressors or compounds. 
56 The dramatic expansion of the OoC field in the past decade has been made possible by the

57 convergence of multiple previously disparate technologies, including induced pluripotent stem 58 cells (iPSCs) and mixed cell culture capabilties, genome editing, 3D printing, sophisticated cell 59 sensors, microfluidics, and microfabrication engineering, which led to the demonstration that 60 dynamic culture conditions significantly influence the physiological maturation and function of 61 in vitro systems. Tissue chips offer promise in, for example, modeling multiple organs and 62 tissues from individual donors of both healthy and disease dispositions, and investigating the 63 responses of these tissues to environmental perturbations and therapeutics with known or 64 unknown mechanisms of action. Worldwide investment from scientific funding bodies (Box 1) 65 has enabled the development of a multitude of 3D tissue models, from relatively simple single 66 cell type organoids to complex multi-cell type, multi-organ microfluidically-integrated systems 67 (Table 1). Consortia, committees and workshops have emerged in Europe, the US and Asia to 68 discuss state-of-the-science aspects of OoCs (Box 1).

In this Review, we will cover how OoCs have evolved over the last decade into a potentially 71 transformational translational science paradigm. OoCs could impact drug discovery and 72 development by offering novel tools for disease modeling and understanding, as well as 73 providing alternative - and potentially more predictive - methods for assessment of toxicity 74 and efficacy of promising new compounds and therapeutics. There are clear opportunities for 75 this technology to provide more rapid, cost-effective, and accurate information on human 76 diseases and drugs being developed to treat them, providing insights for academic, 77 biopharmaceutical, and regulatory scientists that were previously not possible. We will explain 78 how OoCs can model healthy and diseased phenotypes and discuss the promise of linked 79 platforms for the creation of "body on chip" systems. Importantly, we will cover the limitations 80 of OoCs and discuss how defining the context of use of OoC platforms is critical for their 81 continued development. Current considerations and challenges will be detailed, and our 82 predictions for the ongoing era of tissue chip research presented. 
85 OoCs are bioengineered microdevices that recapitulate key functional aspects of organs and tissues. While there is wide diversity in the specific designs of each platform, OoCs range from

87 devices the size of a USB thumb drive to larger systems that reflect multiple linked organs within the footprint of a standard 96-well laboratory plate. All OoC platforms have three critical and defining characteristics: the three-dimensional nature and arrangements of the tissues on the platforms; the presence and integration of multiple cell types to reflect a more physiological balance of cells (such as parenchymal, stromal, vascular and immune cells); and

92 the presence of biomechanical forces relevant to the tissue being modeled (such as stretch 93 forces for lung tissues or hemodynamic shear forces for vascular tissues). One way that 94 biomechanical forces can be introduced to model fluid flow across the tissues is to include 95 microfluidic channels in the systems to deliver and remove cell culture media, and remove associated cell metabolites and detritus. Organoids - another type of multi-cellular 3D tissue model replicating some aspects of in vivo organ structure and function - are not classified as OoCs due to their production through stochastic self-organization (rather than specific cell seeding and growth protocols) and lack of cytoarchitectural structure (rather than provision of scaffolding or specially-shaped culture chambers) ${ }^{9}$.

Table 1 highlights some specifics of how OoCs differ from two-dimensional cell cultures. Each platform design, from 2D plates to complex 3D engineered systems, has advantages and disadvantages. Therefore, the selection of a particular platform will depend on the context of its use, such as the characteristics of the assays and their readouts. One key advantage for OoC platforms is the ability to control cellular and specific tissue architecture to emulate chemical gradients and biomechanical forces. This allows precision control over the biochemical and cellular milieu to model in vivo-like environments and responses. Other advantages include the ability to vascularize or perfuse tissues, either with inclusion of self-assembling endothelial cells that form perfusable lumens, or by use of microfluidic channels that act as engineered

111 vasculature, bringing nutrients and fluidic flow to cells within culture chambers. Also, the ability 112 to incorporate real-time tissue function sensors such as microelectrodes or optical microscopy 
113 markers (for example fluorescent biomarkers) allows for monitoring cell health and activity.

114 Figure 2 illustrates some of the diversity of OoC systems and shows how they can provide a 115 wide range of data outcomes that can be employed during drug development.

\section{7 [H3] Common considerations and challenges}

118 Before OoC platforms are implemented, careful consideration of a large number of variables 119 and challenges is needed to create and validate systems that reflect the context of use and 120 desired outcomes. Although not mututally exclusive, these challenges can be categorised as 121 either biological and technical.

124 [H3] Defining context of use: When creating OoC systems, bioengineers are essentially reverseengineering human cellular systems; that is, taking apart and analyzing the components of the

126 biological system, identifying the key aspects and components needed for function, and using 127 these findings to reconstitute the functional system ${ }^{10}$. Reverse-engineering human tissues and 128 physiological systems is complicated due to an often-incomplete understanding of the 129 composition and interplay of any given tissue and system. Therefore, rather than attempt to 130 comprehensively model a complex system, it may be more useful to engineer simple tissues 131 that can still give relevant and useful answers for the specific field of study. For example, it may 132 be more beneficial to use discrete vascularized brain organoids ${ }^{11-13}$ when modeling 133 glioblastoma, psychiatric disorders or developmental neurotoxicity than to create a complex 134 multi-organ system with cardiovascular, lymphatic and glymphatic components. However, a 135 multi-organ system could provide novel pathological insights into disease mechanisms for 136 disorders or toxicities that require interactions of more than one organ.

138 Currently, OoCs can model certain aspects of a tissue but no single system completely 139 recapitulates a fully functional and integrated human tissue, let alone an organ. Rather, systems 140 are designed to model key aspects of a tissue - or its most characteristic features - to mimic 141 the morphological and functional phenotype of interest; where the phenotype being evaluated 
142 depends on the question being asked. Despite the emerging diversity of OoC platforms (see ${ }^{14}$

143 for a recent review), identifying the base platform choice that can provide answers to the 144 research problem(s) in question remains challenging for end-users.

146 [H3] Cell sourcing: Regardless of system complexity, one universal issue faced by OoC 147 developers and users is renewable cell sourcing (Box 2). Choosing the appropriate cells for a 148 system is partly based on the context-of-use of the platform but also often based on the 149 availability of a particular cell source from commercial entities or from primary donors, which 150 each have advantages and disadvantages. Increasingly, iPSCs or adult stem cells sourced from 151 mass production of tissue organoids are seen as the answer to the lack of available primary 152 cells $^{15}$, and iPSCs have some compelling advantages. For example, iPSCs offer an almost 153 unlimited source of cells, and generating isogenic cell lines from them means that all tissues in 154 multi-OoC platforms could be from the same donor ${ }^{16,17}$, thereby addressing a key source of 155 variability. However, to date, the phenotype of many iPSC-derived differentiated cells such as 156 cardiomyocytes is immature, and protocols for differentiation and maturation are non157 standardized and can be difficult to reproduce (Box 2).

[H3] Cell scaffolds: In addition to understanding a tissue's composition, engineering a tissue requires understanding the functional interplay of cell types and the effect of the scaffold or 161 extracellular matrix [G] (ECM) on the function of the cellular architecture ${ }^{18}$. OoCs may use 162 decellularized scaffolds or seed cells within natural or synthetic hydrogels [G] to create an 163 environment conducive to cell growth, but the ECM composition and three-dimensional arrangement affect cell survival, morphology and polarity ${ }^{19-21}$ and so must be carefully chosen 165 and engineered to promote the formation of appropriate tissue characteristics. The choice of the ECM material must be considered - hydrogels (networks of polymers that swell with water 167 application) are a widely used material due to their biocompatibility, support for cell adhesion, and similarities to many soft tissues and in vivo ECM, but may be difficult to engineer and lack 169 standardized protocols for creation. The complexities of modeling even relatively simple tissues with few cell types can be exponentially magnified when including vascularization, innate or 
171 adaptive immune responses, and the frequent and often large variability in tissue sources

172 between donors/suppliers/batches. Recent advances in bioengineering allow new possibilities

173 for incorporation of biosensors into systems via the ECM. For example, incorporation of

174 fluorescent microgels containing peptides that are cleaved in the presence of specific

175 enzymes ${ }^{22}$ offers the opportunity to use ECM for real-time readouts of OoC assays.

177 [H3] Linking multiple platforms: Linking multiple OoCs into multi-organ systems is not trivial and 178 requires consideration of aspects such as biological (allometric) scaling, maintenance of sterility 179 when building or connecting tissue modules, use of a common medium, incorporation of 180 bubble traps, and control of varying flow rates ${ }^{23,24}$. Additionally, a number of organs and tissues 181 are necessarily missing from even the most complex series of linked OoCs, necessitating the 182 need to account for missing organs. For example, how can a linked platform model important 183 diurnal or endocrine fluctuations - which affect cell and drug metabolism ${ }^{25,26}$ - if tissues 184 producing or responding to those cues are absent? One solution has been the creation of 185 complex engineered 'microformulators' to formulate, deliver and remove culture medium at 186 defined time intervals, simulating the function of missing organ $(s)^{27}$. However, this remains an 187 ongoing challenge.

[H3] Universal medium: Each tissue requires an adequate supply of specific nutrients and 190 growth factors relevant for that tissue, so for linked OoC tissue systems, a key challenge is 191 providing this kind of universal cell culture medium or "blood mimetic". So far, approaches to 192 address this issue have included scaling mixtures of culture media and engineering endothelial 193 barriers. For example, circulating a 50:50 mix of liver-specific and kidney-specific media in a 194 linked liver-kidney system recently enabled the nephrotoxic metabolites of aristolochic acid to 195 be determined ${ }^{28}$. However, as the number of linked systems increases, the success of the 196 scaling solution decreases, as every tissue ends up with a suboptimal culture medium, which 197 will impact the function and therefore physiological relevance of the system. Approaches for 198 linking systems may involve: creating single-pass or recirculating systems of culture medium 199 that can be replenished or modified over time ${ }^{29,30}$; or engineering platforms that allow culture 
200 of tissues in individual modules but provide access to a circulating 'blood surrogate' medium by 201 inclusion of synthetic or endothelial barriers between tissue modules and the circulating 202 medium ${ }^{31-33}$. Some researchers have approached the universal medium problem by providing 203 tissues with appropriate individual support through variation of the surface chemistry of the 204 platform or scaffold on which cells are cultured (e.g. by silanes), while circulating a general serum-free medium to introduce fluidic flow to the system ${ }^{34,35}$.

\section{[H2] Technical considerations and challenges}

208 [H3] Platform design: The characteristics of the assays that are intended to be run on an OoC 209 must be considered early in the design phase or when choosing a particular platform. Many 210 chips incorporate microfluidics, which can supply tissues with the nutrients and factors needed 211 for function and introduce important biomechanical forces such as the shear forces 212 experienced by cells adjacent to vasculature. However, microfluidic designs must carefully 213 model the resulting forces on the tissues because channel diameters, corners, and input/output 214 ports can influence flow rate and therefore tissue performance ${ }^{36}$. Ports for inflow and outflow 215 must be designed to maintain the sterility needed for cell culture while still allowing for culture 216 changes. Also, 'bubble traps' may need to be incorporated, as a bubble in a microfluidic channel 217 can completely block all flow ${ }^{37}$.

219 Modeling biomechanical forces is appropriate in certain tissues; for example, stretch forces for 220 lung alveolar tissues ${ }^{38}$. An elegant solution from an early lung-on-a-chip introduced vacuum 221 channels running alongside a porous membrane onto which lung alveolar cells were seeded on 222 one side and lung endothelial cells on the other. Rhythmic application of the vacuum caused 223 stretching and relaxation of the cell-lined membrane and mimicked the biomechanical forces 224 associated with breathing ${ }^{8}$. This design has been adapted for many other tissues including 225 gut $^{39}$, heart ${ }^{40}$, blood-brain barrier ${ }^{41}$ and kidney glomerulus ${ }^{42}$, highlighting how a simple design 226 concept can be useful for multiple applications.

228 The assays of interest for each platform will ultimately dictate platform design. For example, 229 chips replicating cardiac function likely need to allow access by a microscope and be fabricated 
230 of optically clear materials to allow imaging of cardiac twitching ${ }^{43,44}$. Liver chips modeling 231 oxygen zonation may make use of microfluidic flow rates to create differing zones of oxygen 232 saturation ${ }^{45}$. Neural or muscular (cardiac or skeletal) platforms should incorporate multi233 electrode arrays [G] , or more microscale assays such as patch clamping or voltage clamping to 234 provide readouts of cell activity ${ }^{40}$. Inclusion of biosensors such as fluorophores can allow real235 time readouts of cell function; for example, metabolism, activity, or activation of certain 236 molecular pathways ${ }^{46}$. A recent automated multi-tissue organ system integrated an impressive 237 array of on-chip sensors including electrochemically activated immunobiosensors attached to 238 physical microelectrodes, mini-microscopes, in addition to optical $\mathrm{pH}$, oxygen and temperature 239 monitors ${ }^{47}$. This technical feat highlights the ongoing engineering advances that are enabling 240 real-time non-invasive monitoring of OoC microenvironments.

242 [H3] Platform fabrication: Although hydrogels and other scaffolds can help structure the 243 internal cellular architecture of an OoC, the fabrication materials for the chip itself must be 244 carefully considered. Every material for platform fabrication has a surface chemistry that affects 245 how cells, fluids and compounds bind or absorb into the material. For example, 246 polydimethylsiloxane (PDMS) is a silicon-based organic polymer that is widely used for platform 247 fabrication because it is affordable and easy to work with via soft lithography methods, 248 allowing for fast prototyping and easy iterative design change, and it creates flexible, 249 biocompatible, optically clear platforms that allow modeling of biomechanical forces and real250 time tissue imaging. However, PDMS is gas permeable (which can be an advantage or 251 otherwise) and has a high absorbance for small hydrophobic molecules ${ }^{48}$. Therefore, PDMS 252 becomes problematic for drug studies as the PDMS-based platform itself can absorb a large 253 amount of the drug, or the resulting factors released from the cells may be leached from the 254 effluent. There is also a risk of cross-contamination for chambers or channels adjacent to each 255 other. So, mitigatory approaches for PDMS OoCs include treatment or coating of the polymer256 based surfaces of the device to prevent cell adhesion or drug loss ${ }^{49-52}$. Alternative materials for 257 chip fabrication include glass, silicon, and thermoplastics such as cyclic olefin coplastic (COC) 258 and poly(methyl) methacrolate (PMMA), with the material choice often being a trade-off 
259 between the needs of the platform versus the availability, affordability or fabrication feasibility

260 of the materials.

262 Regardless of fabrication material choice, all OoC platforms require careful characterization of 263 adsorption/absorption profiles. Additionally, the biocompatibility of the materials to be used 264 must be considered and profiled, as unexpected toxicities could appear when repurposing 265 materials for platform fabrication ${ }^{53}$.

268 Toxicity and unknown safety of exposure to human tissues are large sources of failures of 269 potential drug candidates, and accounted for $40 \%$ of losses based on failure data from four 270 large pharmaceutical companies ${ }^{5}$. Traditionally, key individual tissues that are targeted for

271 toxicity assessments include liver, heart, kidney, vasculature, and brain. Methods of assessing 272 toxicity in these organs often use high-throughput but simple cell culture assays, which cannot 273 replicate a complex systemic response to a compound, or animals, which can model complex 274 responses but may not provide an accurate prediction of effects in humans. 275 Pharmacokinetic/pharmacodynamic (PK/PD) modelling [G] and physiologically-based 276 pharmacokinetic (PBPK) modeling [G] can be used to predict the absorption, distribution, 277 metabolism and excretion (ADME) of chemical substances in the body. However, these 278 modeling methods rely on data from other model systems and detailed anatomical and 279 physiological information where it is available. Animal studies are crucial for studying systemic 280 and longer-term effects in full biological systems, but the similarities and differences in 281 comparative physiology to humans can be anywhere on the spectrum between directly 282 translational to confounding or even completely unknown. Indeed, extreme and sometimes 283 tragic examples of the difficulty in translating from animals to humans can be seen in high 284 profile phase I clinical trial failures, although these events are thankfully rare ${ }^{54,55}$. These failures 285 were seen either during the 'first-in-human' phase ${ }^{54}$ or during the dose escalation phase. The 286 drawbacks of current toxicity profiling highlight the intricacies of the translational process from 287 cell culture, to animals, and ultimately to humans, which can place clinical trial volunteers at 
288 high-risk however carefully planned and executed a trial is. Additionally, there is a growing need 289 to predict the toxicity of novel modalities such as biologics, oligonucleotides and large 290 molecules ( $\mathrm{MW}>\sim 900 \mathrm{Da}$ ) that are challenging or impossible to assess in standard animal 291 models. OoCs may have advantages for these modality-specific assessments by allowing 292 modeling of complex human responses in tightly-controlled in vitro systems that may be linked 293 to model organ crosstalk ${ }^{56}$ and can be designed for specific contexts of use ${ }^{57}$.

Single-tissue OoCs offer an alternative way to approach toxicity assessments of potential 296 compounds in various complex human 3D tissues ${ }^{58}$. In 2D liver cultures, hepatic cell line 297 cultures poorly represent primary human hepatocytes ${ }^{59}$, and the latter cells rapidly de298 differentiate over 24 hours ${ }^{60}$, limiting their usefulness in evaluating either subacute or chronic 299 exposure effects and systemic toxicities. An example of how OoCs could address such issues is a 300 recently developed 3D liver OoC system that can maintain healthy cell cultures for over 28 days 301 (Table 2) and mimic the in vivo environment of the liver (to include hemodynamic flow, oxygen 302 zonation and inclusion of immune components ${ }^{61,62}$, which opens new pathways for 303 ADME/toxicity studies. Oxygen zonation in this liver platform was achieved by controlling the 304 flow rate of medium through the platform to create zones of differing oxygen tension, and 305 coupling computational modeling of this tension to direct temporal and spatial monitoring of 306 oxygen-sensitive dyes in the system ${ }^{45}$. This highlights how use of biomechanical forces and 307 direct experimental assays from real-time biosensor readouts can be combined to provide 308 powerful tools for accurate replication of clinically-relevant toxicity profiles. Separation of the 309 sinusoid (vascular channel) and hepatic compartment by a porous membrane allows 310 physiologically-relevant addition of drugs, immune cells and other factors to the model ${ }^{62}$. 311 Another recent study comparing a liver on a chip from rat, dog and human cell sources 312 elegantly showed species-specific differences in hepatotoxicity, highlighting the importance of 313 using human-specific cells for certain assays, while confirming the validity of the use of non314 human models for others ${ }^{63}$ (Table 2). 
316 For the heart, which is another important target organ of toxicity, a number of heart-on-a-chip 317 systems have been developed that model the complex matrices of cardiomyocytes, (cardiac) 318 fibroblasts, endothelial cells and vasculature that interact in vivo in a highly ordered manner, 319 which can be easily perturbed by drugs, drug-drug interactions, or off-target side effects. Since 320 in vitro screens are now an integral part of drug development to characterize cardiac safety 321 liabilities, the current heart-on-a-chip systems are useful as they model human responses to 322 injury (Table 2), and show appropriately aligned sarcomeres, rhythmically synchronized beating 323 patterns, and physiologically relevant resting membrane potentials ${ }^{44,64-67}$. Other structures in 324 the heart, such as cardiac valves, have been bioengineered to assess the off-target cardiac side 325 effects of dopamine/serotonin production/reuptake influencing-drugs, such as pergolide, which 326 are used in clinical treatment for psychiatric disorders such as Parkinson's disease ${ }^{68}$. However, a 327 large problem with all cardiac OoC systems currently using iPSC-derived tissues is the fetal 328 phenotype of most resulting cardiomyocytes ${ }^{69,70}$. Despite this, recent advances using electrical 329 and mechanical stimulation to 'train' the developing cells or cardiac "organoid" growth in fatty 330 acid-based culture medium and inclusion of other relevant cell types seems to encourage a 331 significantly more mature phenotype ${ }^{71-74}$, further expanding the potential use for OoC in the 332 cardiotoxicity field.

334 Other important tissues for toxicity profiling include those from the kidney, gut, and lung. 335 Developmental toxicity assays, including neurotoxicity, are also relevant for many exposure 336 studies. OoC models of the kidney (nephron and proximal tubules) can be used to model 337 readouts relevant for nephrotoxicity profiling such as filtration, reabsorption, transport of 338 various molecules, and action of protein transporters ${ }^{75-78}$. Indeed, a kidney-on-a-chip system 339 was used to elucidate that polymyxin-B nephrotoxicity may be caused by the cholesterol 340 biosynthesis pathway, highlighting how OoCs could not only be used to test the safety of novel 341 chemical molecules but also shed light on toxicological pathways of FDA-approved molecules ${ }^{78}$ 342 (Table 2). Gut-on-chip systems can model certain aspects of the bioavailability and activity of 343 drugs, by creating in vitro intestinal epithelia and exposing these tissues to relevant 344 biomechanical forces, such as flow and peristalsis ${ }^{79,80}$. Inclusion of immune and microbiome 
345 factors become critical for true human relevance, both of which by themselves are huge areas 346 of research, although there is progress being made in inclusion of these in both organoid ${ }^{81}$ and 347 microfluidic systems ${ }^{82-85}$. For example, the "HuMix" model to recreate human-microbial 348 crosstalk allows researchers to investigate the causal relationships between the gastrointestinal 349 microbiota and certain human diseases, but could also be used in toxicology and 350 pharmacokinetic studies ${ }^{82}$. Toxicity profiling of inhaled substances can benefit from lung-on-a351 chip models that can recapitulate the air-liquid interface of the lung alveoli ${ }^{8,86}$ and model 352 effects such as exposure to bacteria, drug-induced pulmonary edema and cigarette smoke ${ }^{87}$. 353 Developmental neurotoxicity can be modeled in platforms containing 3D neural tissues. For 354 example, in a study that used RNA-Seq readouts from neural constructs exposed to 60 drugs of 355 known toxicity, a predictive model based on linear support vector machines had over $90 \%$ 356 accuracy in predicting the toxicological impact of 'blinded unknown' compounds ${ }^{13}$, highlighting 357 the potential power of these types of 3D models for predictive toxicology. Other developmental 358 toxicological vulnerabilities have been assessed using placenta-on-a-chip models that can 359 recapitulate the ability of compounds to cross or affect the maternal-fetal barrier ${ }^{88,89}$. 360 Readouts of vascular-related toxicity may be critical for therapeutics, and vascular networks on 361 OoCs have been used to investigate vascular toxicity with chemotherapeutics ${ }^{29,90}$, and risk 362 factors for complications such as thrombosis from monoclonal antibody treatments ${ }^{91}$.

364 Finally, linked multi-organ systems could expand OoC applications into organ interactions and 365 systemic toxicity profiling, and these are discussed further in section 6.

\section{[H1] Disease modeling on a chip}

368 In addition to being useful as tools for understanding toxicity in human tissues, OoCs also offer 369 ways to model disease states in vitro, thereby allowing mechanistic investigation not only of 370 disease pathologies but also of the efficacy and potential off-target effects of therapeutic 371 interventions. The potential enhanced understanding of human disease physiology from 372 modeling diseases on OoCs could help address the high attrition rates of promising compounds 373 seen during both lead optimization and clinical development stages due to lack of efficacy ${ }^{5,92}$. 


\section{[H2] Stem cells and tissue chips - powerful partners}

376 While many OoCs have been developed to model disease phenotypes using primary or cell line 377 sources, the increasing use of iPSCs, plus the novel option of using the mass production of organoid technology as a way to source adult stem cells in biomedical research, has also led to 379 the increased development of an array of diseases-on-chips including: cardiac (atrial and ventricular) myopathies ${ }^{72,93,94}$; asthma ${ }^{95}$; vascular abnormalities ${ }^{96}$; polycystic kidney disorders ${ }^{97}$;

381 as well as neural disorders - including ones mimicking aspects of neurodegenerative and psychiatric disorder phenotypes ${ }^{98,99}$ - and rare pediatric diseases such as Hutchinson-Gilford

383 Progeria Syndrome ${ }^{100}$. However, a limitation associated with using stem cell-derived cells in 384 OoCs include difficulties in producing an adequate number of mature, differentiated cells with 385 the necessary purity for many tissues (for more see Box 2).

387 Despite these current limitations, one early example of the power of iPSCs' use in OoCs, 388 coupled with genome editing technologies, investigated the rare childhood pediatric cardiomyopathy Barth Syndrome. Stem cell derived-cardiac tissues from patient donors were created and modeled on 'muscular thin films', which replicated the disordered sarcomeric organization and weak contraction properties seen in the disease ${ }^{101}$. Using genome editing techniques to 'correct' the faulty TAZ gene in the iPSC-derived cardiomyocytes, mitochondrial abnormalities underlying the disease were identified. These results highlight the potential use of OoCs as models for the critical stages of target validation where the creation of multiple tissue types from the same patient, and the generation of isogenic control tissues by genetic editing methods for any number of genetically-based diseases, can enable detailed and specific mechanistic studies for these disorders ${ }^{102}$.

[H2] "You-on-a-chip" for common and rare diseases

400 Disease modeling on OoCs could contribute to the development of precision medicine. OoCs 401 modeling angiogenesis ${ }^{103}$, tumor growth $^{104}$, and intra- and extravasation ${ }^{105,106}$, have all 402 contributed to the development of vascularized and metastatic breast cancer models ${ }^{107-110}$. The 403 treatment of patient-derived tumors on chips with chemotherapeutics enabled treatment 
404 comparison and optimization ${ }^{108}$, which is a step towards using this technology for precision 405 medicine. Tumor-on-a-chip platforms have also helped parse out the mechanistic effects of 406 different chemotherapeutic agents on the resulting 'microtumors' ${ }^{90}$. Other tumor-on-a-chip 407 models include neural glioblastoma ${ }^{111}$, renal cell carcinoma ${ }^{112}$, as well as lung ${ }^{113}$, pancreatic ${ }^{114}$, 408 colorectal $^{115}$, ovarian ${ }^{116}$, prostate ${ }^{117}$, and cervical ${ }^{118}$ cancer, among many other types.

410 While many of these models were created with cancer cell lines, an obvious and powerful 411 opportunity arises when patient-derived primary or iPSC-derivatives are seeded onto OoC 412 models, creating "patient-on-a-chip" models. This could inform the stratification of cancer 413 patient populations into subpopulations that respond optimally to different chemotherapeutic 414 regimens or cocktails, but could also lead to development of "you-on-a-chip" for rare cancer 415 patients or those with unusual etiologies. Communities with rare diseases could benefit 416 tremendously from the opportunity to recreate these pathologies on chips (see ${ }^{119}$ for a 417 review). For example, patient-derived pancreatic ductal epithelial cells can be used to create a 418 pancreas-on-a-chip to potentially understand the cystic fibrosis transmembrane conductance 419 regulator protein and its role in insulin secretion ${ }^{120}$. If iPSC protocols become available for 420 pancreatic cell creation - a current challenge with promising progress in the field ${ }^{121}$ - then 421 modeling of an individual with cystic fibrosis on a chip becomes possible, which could prove 422 useful to understand the high risk of diabetes and glucose imbalance in this population.

425 Both OoC and organoid 3D models have strengths and limitations (Table 1), but innovative ways 426 to combine the technologies and introduce related ones such as 3D bioprinting - so-called 427 'synergistic engineering ${ }^{122}$ - adopts strengths from multiple 3D bioengineering fields to create 428 reliable predictive tissue models with the opportunities for higher throughput screening (see ${ }^{123}$ 429 for a comprehensive review). For example, both organoids (which self-organize into three 430 dimensions) and bioprinted tissues (where cells are deposited in a specific manner) can be 431 seeded or printed in multi-well plates with media flow and inclusion of other biomechanical 432 forces, creating platforms with multi-tissue components that are amenable to larger scale 433 commercial production. An example of these combined technologies includes vascularized 
434 organ 'buds' that can be perfused by a common medium ${ }^{124}$ and bioprinting of endothelialized 435 myocardium in a microfluidic perfusion bioreactor ${ }^{125}$. In the case of the latter, multiple 436 bioengineering techniques were combined to create an innovative tool for predicting 437 cardiovascular toxicity. First, endothelial cells were encapsulated into bioprinted microlattices 438 to allow formation of an endothelial vascular bed, after which cardiomyocytes were introduced 439 forming a myocardial tissue with good alignment to the bioprinted vascular bed. Finally, 440 inclusion of the tissue construct into a microfluidic bioreactor allowed continuous vascular 441 perfusion and real-time monitoring of cardiac contraction phenotypes for up to 2 weeks.

443 As with all disease models, the demonstration that these 3D tissue models effectively mimic the 444 behaviors of the disease, as well as the responses to therapeutic drugs, in vivo is critical for 445 their validation.

[H1] 6. Creating a "Body on a Chip"

448 Linkage of multi-organ tissue systems is of clear benefit to model complex organ-organ 449 interactions and inform PK/PD and PBPK modeling, ADME profiling, and quantitative systems pharmacology (QSP) and other computational modeling. Over the last decade, many efforts have been undertaken to integrate multiple systems and overcome the challenges associated

452 with this (see ${ }^{126}$ for a review). Indeed, US governmental funding from the Defense Advanced 453 Research Project Agency (DARPA) was specifically allocated to create and link 10 organ systems 454 (see Related links) that were viable for 28 days into a single 'body on a chip' as part of broader 455 efforts by the US National Institutes of Health (NIH), FDA and DARPA to fund the development 456 of tissue chips to advance regulatory sciences (see Related links). From this funding, two recent 457 publications showed how a 10-organ "physiome on a chip" combined with QSP computational 458 approaches could model distribution of in vitro pharmacokinetics and endogenously produced 459 molecules ${ }^{127}$; and how a robotic 'interrogator' maintained the viability and organ-specific 460 functions of eight vascularized, two-channel organ chips (intestine, liver, kidney, heart, lung, 461 skin, blood-brain barrier and brain) for 3 weeks in culture ${ }^{128}$. 
463 The study of prodrugs ${ }^{129}$, which are metabolized by the body from inactive to active 464 compounds, could benefit, as could the development of novel compounds which that rely on 465 (or cause) bioactivation ${ }^{130}$. Slow release mechanisms (e.g. slow-release painkillers and 466 contraceptive injections or implants), or compounds produced by non-traditional methods such 467 as synthetic biology or genetic engineering, could also be extensively assayed for unexpected 468 side effects. Coupling these types of new molecular technologies with powerful computational 469 modeling tools, including quantitative systems pharmacology (QSP) ${ }^{131}$, machine learning ${ }^{13}$, and 470 artificial intelligence $(\mathrm{Al})^{132}$, could offer novel and helpful insights for current toxicological 471 assessment. For example, capecitabine and tegafur (anticancer prodrugs) have been shown to 472 be effective in a multi-organ pneumatic pressure-driven platform ${ }^{133}$, and recently Boos et al ${ }^{134}$ 473 used a hanging-drop organoid system to test how products metabolized by human liver 474 microtissues affect embryoid bodies. The prodrug cyclophosphamide (activated by cytochrome 475 P450) was added to the system and a 50\% drop of embryoid differentiation seen, 476 demonstrating how powerful synergistically engineered microfluidic systems can be not only 477 for prodrug investigation, but also embryotoxicity in this case.

479 Challenges with linking systems include how to: scale the organs of interest (e.g. allometrically, 480 based on body size, or metabolically ${ }^{24}$ ); model fluid flow dynamically through the system and 481 scale flow appropriately for each tissue ${ }^{23}$; supply all tissues with adequate growth factors and 482 culture medium support (for example via a blood surrogate culture medium ${ }^{7}$ or by separation 483 of cultures by endothelial barriers ${ }^{135}$ ); and design and fabricate these complex systems. One 484 approach to linking systems that avoids many challenges faced with physically linking organ 485 cultures involves functional coupling such as running media through physically separate 486 systems sequentially to model multi-organ ADME. In the case of Vernetti et al $^{136}$, this approach 487 showed that organ-specific processing of the tested compounds was consistent with clinical 488 data, and additionally uncovered that a liver-bioactivated microbiome metabolite crosses the 489 blood-brain barrier using a neurovascular unit $\mathrm{OoC}^{137,138}$. 
491 A number of physically linked systems via microfluidics and pneumatic or peristaltic pump

492 mechanisms have been published (Figure 3) and include systems that have revealed, for 493 example, novel mechanisms of aristolochic acid nephrotoxicity ${ }^{28}$, the metabolic coupling of 494 endothelial and neuronal cells in the neurovascular unit ${ }^{139}$, and inflammatory crosstalk between 495 the gut and liver ${ }^{140}$. For example, Chen et al ${ }^{140}$ examined an integrated gut-liver transwell OoC 496 and showed that modulation of bile acid metabolism was seen in the linked system. 497 Meanwhile, in an inflammatory state (modeling endotoxemia by increasing circulating 498 lipopolysaccharide levels), hepatic biotransformation and detoxification pathways showed 499 changes, highlighting that even relatively simple OoC models can give valuable information on 500 organ interactions.

501

502 Additionally, a number of multi-organ systems demonstrating utility in toxicology and disease 503 modeling applications are appearing in the literature, including systems modeling homeostatic 504 mechanisms ${ }^{32,141}$, hepatic metabolism and off-target cardiotoxicity ${ }^{34,142}$, and the female 505 reproductive tract and menstrual cycle ${ }^{143}$ that reproduced a 28 day hormonal cycle in a 506 platform including ovarian tissue, fallopian tube, uterus and cervix, but also included a liver 507 module for reproductive toxicology utility (Figure 3A). Synergistically engineered multi-tissue 508 organoid-based platforms linked by microfluidics are also joining the expanding cadre of multi509 organ OoC tools ${ }^{47,133,144,145}$. Importantly, many of these systems incorporate a variety of real510 time assays and biosensors for ongoing cell health and function readouts and can support 511 extended cell culture (<28 days), allowing chronic and repeated testing of compounds for 512 systemic toxicity evaluation ${ }^{35,146}$. Some of these linked systems are becoming more broadly 513 available to researchers either through contract research organization (CRO)-based services or 514 purchase of off-the-shelf systems, although the latter are generally simpler organoid-based 515 higher throughput multi-well plate systems. Manufacturing the more complex OoC systems 516 designed by engineering labs is still an obstacle to widespread implementation in biomedical 517 labs. 
520 As OoCs become increasingly commercially available, reproducibility of the technology at

521 multiple sites is becoming critically important. Negotiating legal frameworks to facilitate sharing

522 of proprietary information and technologies between organizations can can be lengthy.

523 Meanwhile, sometimes critical exchange of reagents and trained personnel can become costly,

524 and unexpected obstacles can emerge from simple processes such as shipping cells and

525 resources. Some questions that arise from these obstacles include: should cells be shipped in

526 differentiated or undifferentiated forms? Should platforms be seeded with cells, or should the

527 recipient fabricate the systems from shared molds instead? Can cells be shipped in OoC plates

528 in a frozen state and simply thawed prior to use by end-users? Thorough consideration of the 529 most straightforward processes can become complex and expensive.

[H2] Robust, reproducible, reliable platforms

532 The US government has provided almost a decade of support for OoC development, and 533 although the DARPA 'body-on-a-chip' program has now ended other federal agencies continue 534 to support US-based $\mathrm{OoC}$ development, and agencies in Europe and elsewhere are also 535 supporting OoCs (Box 1). In particular, the National Center for Advancing Translational Sciences 536 (NCATS) has created two new programs since 2016 that focus on creation of reproducible, 537 reliable, and automated systems that are accessible to the wider community. The Tissue Chip 538 Testing Centers (see Related links) initiative began in 2016 to support two independent centres 539 charged with onboarding developers' tissue chips, monitoring reproducibility of assays and 540 outcomes, and investigating additional parameters that are of use to the community. The first 541 publication addressing independent validation of a kidney proximal tubule model was recently 542 published $^{147}$ and a number more are forthcoming. To encourage the development of robust 543 automated systems with smaller laboratory benchtop footprints, the NCATS Tissue Chips in

544 Space program also promises advances for the technical development in the field (Box 1). These 545 programs, plus commercial pressures, are pushing the move towards more 'turn-key' OoCs to 546 help reduce or remove the need for the specialized infrastructure and highly-skilled personnel, 547 which is currently often required for OoC implementation. 
$550[\mathrm{H} 3]$ Increasing throughput: Most complex non-organoid tissue chips are currently very low 551 throughput, where only dozens of replicates (at most) can be performed at any one time. 552 Consequently, during the early stages of drug discovery, at which many thousands of potential 553 hits can be identified in a short time-frame through standard high-throughput screening assays, 554 the use of such chips is likely to be considered cost- and time-prohibitive for pharmaceutical 555 companies at present. Technological advances to create more automated, miniaturized OoC 556 systems that can become 'turn-key' technologies for facile use will be crucial to increasing 557 throughput and the number of replicates per platform.

559 [H3] Scaling up of reliable manufacturing processes: One difficulty with many OoCs is how to 560 scale-up system manufacturing to an industrial pace. Most early OoC designs are bespoke and 561 fabricated in-house at the developers' institutions, where fabrication is limited by cost and 562 availability of both manufacturing equipment and personnel. Therefore, academic laboratories 563 should focus on early quality control of the chips produced in-house, to ensure reliability and 564 reproducibility before scale-up can occur. This means careful compilation of standard operating 565 procedures for chip design and creation, and designing clear quality control procedures that can 566 be easily followed at other laboratories or manufacturers. Since most academic laboratories are 567 not equipped for scale-up of production, the creation of spin-off or start-up companies, or 568 formation of partnerships with manufacturing firms to mass-produce chips, becomes 569 necessary. At this stage, it would be extremely useful for all manufacturers to conform to $\underline{\text { Good }}$ 570 Manufacturing Practice guidelines (see Related links) such as those set forth by the US FDA, 571 which cover issues including equipment verification, process validation, sanitation and 572 cleanliness of manufacturing facilities, and appropriate training of personnel. While this 573 guidance is to ensure the safety and reliability of manufacturing processes for foods, drugs, and 574 devices for medical use, and is therefore not necessary for OoC manufacturing, it would still 575 provide excellent standards for reliability of chip production across all fields and help to broadly 576 increase confidence in the systems. In order to increase end-user confidence in the reliability 577 and fidelity of mass-produced platforms, additional considerations should be taken that all 578 biological assays are created on chips under Good Laboratory Practices, as this is critical for 
579 preclinical toxicology testing and has been identified as a major reason for drug development 580 attrition rates ${ }^{148}$. In addition, there is a need for independent "qualification" labs to test OoCs 581 and their usage with available cell types, much like the NCATS Tissue Chip Testing Centers (see 582 Creating a "Body on a Chip") or the European Union Reference Laboratory for Alternatives to 583 Animal Testing European Centre for the Validation of Alternative - EURL ECVAM (see Related 584 links).

[H3] Onboarding versus outsourcing: Due to the expense and complication of technology 587 transfer for some OoCs, developers may face the decision between supplying a commercial 588 product for purchase to be used independently in a customer's laboratory, or offering services 589 through a CRO to OoC consumers. If researchers decide to commercialize their OoC platforms, 590 technology transfer and onboarding processes should become seamless, reliable and 591 standardized for every customer. Meanwhile, retaining the personnel, infrastructure and 592 resources necessary for OoC use within a CRO-based service means customers should expect 593 high standards of the research produced. However, the flexibility and adaptation of the chips 594 for specific contexts of use may be limited because CROs may not offer particular assays or 595 services. As this burgeoning field is still young, many developers and companies are choosing to 596 adopt aspects of both business models. Some offer OoC devices that can be onboarded 597 relatively easily but may need specialized equipment and/or extensive technical support. Other 598 CROs perform experiments in-house in collaboration with academic or industry researchers to 599 help advance continuing R\&D on the system.

601 [H3] Managing expectations: While the potential of OoCs is exciting, the technology is at an 602 early stage, so providing realistic caveats and limitations to potential consumers is critical to 603 avoid overselling its current capabilities. Some challenges faced within the field may be 604 resolved over the next decade or so - issues with cell sourcing will continue to be addressed as 605 the stem cell field matures, for example. Other limitations may take longer to resolve - for 606 example, reduction and refinement of animal use are laudable and achievable aims and are 
607 within the realm of possibility already, but full replacement of animals in drug development is 608 generally seen as unlikely in the near future.

610 One approach to managing expectations has been employed by government funding agencies

611 in the US where creating partnerships between research and regulatory agencies, such as the

$612 \mathrm{NIH}$ and FDA, over the last decade has allowed regulators access to OoC developers and their 613 unpublished data to help inform system development. Conversely, it has enabled researchers 614 to design useful platforms to provide data for regulatory assessment. This has led to familiarity 615 of the technology among the regulatory community in the US, which ultimately can help pave 616 the way for OoC data inclusion in IND (Investigational New Drug) [G] and NDA (New Drug 617 Application) [G] packages in the future.

620 As OoCs continue along a path towards widespread commercialization, validation must be 621 considered. Importantly, the term 'validation' means different things to various stakeholders, 622 but could be considered as involving three stages or principles ${ }^{149}$. First, physiological validation 623 could be defined in the context of 'analytical performance', including addressing features such 624 as sensitivity, specificity and precision (essentially reproducibility). This validation step is 625 necessary to create a tissue chip that appropriately and reliably mimics the tissue of interest 626 and responds in relevant ways to compounds of known action or toxicity, and it should be 627 performed by OoC developers. Second, qualification or validation to show biological in vivo 628 relevance should come next, although there is debate in the field as to whether animal or 629 human responses should be used for this stage. Animal responses are broadly used in current 630 drug development, which supports the argument that they should be the 'gold standard' for 631 OoC responses to be compared against. Conversely, predicting human responses is the aim for 632 the field, which supports the focus on generation of human responses on OoCs. Reproducibility 633 and setting the standards for qualification currently fall under the remit of, for example, the 634 NCATS Tissue Chip Testing Centers. The third stage, industrial validation, or OoC adoption by 635 industry and regulatory agencies, will involve the generation of data from proprietary 636 compounds and submission of that data to regulatory agencies. All of these stages of validation 
637 are currently underway. In the US, the FDA has also partnered with a number of OoC 638 companies to get hands-on experience with $\mathrm{OoC}$ data, as they expect this type of data to be 639 submitted to them in the near future.

Taken together, the three stages/principles of validation/qualification described above will help

642 address international guidelines for novel methods, for example the Organisation for Economic

643 Co-operation and Development (OECD) Guidance Document on the Validation and International

644 Acceptance of New or Updated Test Methods for Hazard Assessment (see Related links) These

645 guidelines describe necessary assay details for validation such as the rationale, the endpoints

646 and limitations, protocols, variability, performance with reference and known chemicals, and

647 comparisons to existing assays. Importantly, the OECD guidelines also state that data 648 supporting the validity of the method must be available for review. To address this need for all 649 stakeholders, the NIH's NCATS also funds an MPS Database, which is tasked with integrating all 650 the data from the Testing Centers, as well as data from a number of other $\mathrm{NIH}$-funded 651 developers, FDA users, and commercial OoC suppliers. This centralized database acts as a public 652 repository for a broad range of $\mathrm{OoC}$ data and will prove useful for developers, industry and 653 regulatory bodies over the coming years, with a recent report highlighting functionality for data 654 visualization, inter- and intra-study reproducibilities and power analyses calculations ${ }^{150}$.

656 Additionally, underpinning the needs of the above validatory steps, the accurate 657 standardization of methodologies used for generating empirical data should be considered. The 658 term 'standardization' brings on new challenges with respect to what 'standardization' means 659 for either technical, analytical or biological aspects of OoCs. So, 'performance standards' should 660 be established for the analytical validation and biological qualification of OoCs. To this end, the 661 deposition of technical, analytical and biological data into the MPS-Database will help set some 662 of the standards, reducing the need for each user to develop their own methodologies, assays 663 and analytical methods. At the same time, many US government-funded researchers are 664 working with regulatory and industrial end-users to evaluate what should be considered 665 accepted metrics that are translatable to other laboratories and applications. 
668 There are multiple stages at which OoC platforms could be implemented in drug discovery and 669 development, and the platform type may differ depending on the stage (see Figure 1). High670 throughput plate-based OoCs with relatively simplistic (but cheap and fast to produce) tissue 671 constructs could prove useful for target identification, lead selection and lead optimization. 672 Low- to medium-throughput $\mathrm{OoC}$ platforms that model more complex tissue-tissue or organ673 organ interactions could be more useful for preclinical single or double organ toxicity and 674 efficacy studies. Multi-organ systems - while perhaps the most complex and expensive to 675 develop - offer promise for reducing the need for animal studies and for use in parallel with 676 phase I and II clinical trials. Finally, OoC platforms from patient stem-cell-derived sources could 677 be used during later clinical trial phases (III and IV) as well, for in vitro therapeutic testing 678 before in vivo administration, or for concurrent monitoring of approved therapeutics. 679 Ultimately, the potential safety and efficacy of a drug or drug candidate could be evaluated 680 using OoCs in generic, or even individualized, human platforms, giving "first-in-human" testing 681 a new connotation.

683 Coupling OoC technology with techniques such as gene editing ${ }^{151}$ (particularly when a series of disease-relevant mutations are introduced onto a single genetic background) offers powerful 685 ways to increase the predictive power of these tools further in disease modeling and toxicology. We also see opportunities to discover and validate clinically-translatable biomarkers by creating 687 datasets to correlate in vitro OoC readouts with clinical outcome measures. For example, using OoCs to produce 'omics'-based (and even real-time) readouts could promote the identification 689 and evaluation of appropriate endpoints surrogate to those in the clinic, which could provide valid and reliable measures of change in human subjects. These endpoints and readouts could 691 be quantified and assessed for clinical benefit and compared to traditional enzymatic, 692 biochemical or histopathological assays, as well as offer ways to assess both short- and long693 term clinical changes. Ultimately, the use of OoC readouts detailing changes in molecular 694 signatures that have been validated against traditional methods and demonstrated clinical 695 relevance could become a common practice in drug development. 
697 In order to help smooth the adoption and implementation of OoCs in the drug development 698 process, continued engagement and discussions with OoC developers and end-users is critical, 699 as is engaging with regulatory bodies. A 2017 report predicted that the global OoC market could 700 grow by $38 \%$ per year to become a US\$117M/year industry in 2022 (based on market analysis 701 by Yole Développement) - with the potential to become a multi-billion dollar industry. In 702 support of this predicted growth and the utility of OoCs at various stages of drug development, 703 a recent anaylsis predicted up to a $26 \%$ reduction in R\&D costs in the pharmaceutical industry 704 by adopting OoC technology ${ }^{152}$, and it is anticipated that OoC data will be included in IND and 705 NDA submissions to the US FDA in the near future.

707 There is optimism that OoC systems may one day outperform traditional models, making the 708 understanding of human diseases and development of drugs to treat them more rapid, 709 efficient, and cost-effective, and in so doing replace, reduce and refine (the " $3 R s^{\prime \prime}$ ) the use of 710 laboratory animals. Nevertheless, much work remains to address the challenges discussed in 711 this article, and thereby determine and realize the potential of this technology. According to 712 the 2018 Gartner report (see Related links) on the hype cycle of emerging technologies, OoCs 713 (referred to as 'biochips' in this report) are now in the 'Peak of Inflated Expectations' phase. 714 Disillusionment and a stall in progress often occurs after this phase because the technology fails 715 to live up to the preliminary, and often inflated, expectations, before the field recovers and 716 productivity resumes, with more modest expectations. Therefore, the aim for emerging 717 technologies is to reach this productive plateau as quickly as possible, when $20-30 \%$ of the 718 potential audience has adopted the innovation. Right now, this is estimated to be 5-10 years for 719 OoCs. It will take the coordinated global efforts of the OoC community to help this technology 720 reach that potential global audience and ultimately, help transform science, medicine, and 721 patients' lives. 


\section{2 [bH1] Box 1: Collaborative tissue chip development efforts}

723 In 2010, the US Food and Drug Administration (FDA) and the US National Institutes of Health

724 (NIH) created a Joint Leadership Council to help speed the translation of biomedical discoveries

725 at the laboratory bench to commercial availability of new therapeutics. Under this mandate,

726 the Advancing Regulatory Science program was initiated, with awards issued to address

727 distinct, high priority areas of regulatory science. Based on the promise from these funded

728 projects, from which the seminal lung-on-a-chip work was published ${ }^{8}$, the NIH and FDA

729 partnered with the Defense Advanced Research Projects Agency (DARPA) to fund two 5-year

730 programs for the development of OoCs. The NIH program, called "Tissue Chips for Drug

731 Screening" (see Related links), awarded funding to develop 3D microsystems to represent

732 multiple tissue types and also concurrently funded a program to explore the use of stem cells

733 and progenitor cells to differentiate into the multiple cell types that would be needed to

734 populate the microsystems. DARPA's MPS program (see Related links) focused on developing a

735 reconfigurable platform of at least 10 human organs or tissues in an integrated system that

736 could mimic and replicate biological crosstalk between tissues. While both initial programs

737 ended in 2017, the NIH continues to offer funding for further development of OoCs in an

738 expanding array of programs, including for disease modeling, inclusion of immune factors,

739 modeling of Alzheimer's Disease, use in the context of clinical trials, and as part of the NIH Help

740 End Addiction Long-term (HEAL) initiative (see Related links) to address the US opioid epidemic.

741

742 The FDA has offered advice and guidance from a regulatory standpoint for the past decade, and

743 recently signed Memorandums of Understanding with a number of commercial tissue chip

744 companies to on-board the technology to FDA laboratories. Additionally, the IQ Consortium

745 (see Related links), a non-profit organization consisting of pharmaceutical and biotechnology

746 company representatives, partnered with US government funding agencies in 2016 to add end-

747 user stakeholder perspectives to the field. The IQ Consortium recently published a series of

748 manuscripts on the characterization and use of $\mathrm{OoC}$ sytems in safety and toxicity profiling 749 applications ${ }^{56,153}$ and for modeling skin $^{154}$, lung ${ }^{155}$, the GI tract ${ }^{156}$, kidney $^{157}$ and liver ${ }^{158}$. 
751 In Europe, the Institute for human Organ and Disease Model Technologies (hDMT, see Related

752 links), headquartered in the Netherlands, leads the way on integrating state-of-the-art human

753 stem cell technologies with biotechnical fields to support the development and validation of

754 human organs and disease models-on-chip. The hDMT consortium helped co-ordinate one of

755 the European Union's Horizon 2020 research and innovation programs termed Organ-on-Chip

756 Development ( $\underline{\text { ORCHID }}$, see Related links), and in late 2018 launched the new European Organ-

757 on-Chip Society (EUROoCS, see Related links) that will encourage development and

758 coordination of tissue chip research in Europe. Other countries are following the hDMT

759 example and are establishing similar organ-on-chip networks in Israel, UK, the Scandinavian

760 countries and Switzerland.

761

762 One key tenet of collaborative partnerships for tissue chip development has been the 763 involvement of different stakeholders to help advance each of their missions. For example, 764 partnership of tissue chip developers with the Comprehensive in vitro Proarrhythmia Assay 765 (CiPA, see Related links) initiative helps provide tools to fulfill CiPA's mission of engineering 766 assays for assessment of the proarrhythmic potential of new drugs with improved specificity 767 compared with current assays, while demonstrating the utility of tissue chips for toxicity 768 screening.

Another collaboration between the NIH and the Center for Advancement of Science in Space

771 (CASIS, see Related links) allows researchers to use the microgravity environment on the

772 International Space Station (ISS) to conduct biomedical research. The program, which partners

773 with the International Space Station National Laboratory (ISS-NL), is using microgravity as a tool

774 to investigate Earth-based disease pathologies such as formation of kidney stones that would

775 otherwise be difficult or take too long to model on Earth. Moreover, researchers and space

776 payload developers work collaboratively to adapt OoC platforms and make them robust enough

777 for rocket launch, spaceflight, integration into ISS facilities, and splash-down. This is leading to

778 advances in the technical engineering of robust platforms capable of higher throughput (>24

779 replicates running concurrently) with a much smaller footprint. The systems are turn-key 
780 enough to be "astronaut-proof", meaning that non-scientist workers (in this case astronauts,

781 most of whom are not trained in laboratory techniques) can perform the necessary

782 interventions - both in space and in the future on Earth in a variety of applications ${ }^{159}$.

783 


\section{4 [bH1] Box 2: Cell sourcing for 3D tissue engineering}

785 The common aphorism of "all models are wrong but some are useful" is apt when considering

786 cell sourcing for microphysiological systems (or any bioengineered tissue models). No cell

787 source is perfect; many have serious caveats; but even the most problematic cell source can

788 provide useful information if used appropriately based on the question being asked. Cells

789 seeded in tissue chips come from three main sources: commercially available cell lines; primary

790 cells from human donors; and induced pluripotent stem cell (iPSC)-derived sources.

792 [bH2] Commercially available cell lines: Cell lines should have extensive validation of purity and 793 viability when received from reliable sources (such as the American Type Culture Collection) 794 and are often proliferative as well as easy to culture and transfect. These cells have clear and 795 reliable culture protocols, generally respond in stable and predictable ways and will likely 796 contribute to high reproducibility. Commercially available cells can be excellent sources of hard797 to-find cell types, or when primary and iPSC sources are unavailable. However, these cell lines 798 are approximations for the primary cell types found in vivo and should be periodically evaluated 799 to see how far from the primary cell phenotype the new generations are straying.

[bH2] Primary cells: The clear advantage of using cells from human donors is that the cells capture the phenotype (presumably genetically and functionally) of the mature adult state. Primary cells can model disease pathologies when sourced from donors with certain diseases and can accurately reflect clinical population variance in their phenotypes. However, because genetic and epigenetic differences arise during a donor's lifetime, variability between donors or batches can be hard to identify and track. For some primary tissues (for example: neural cells), access from donors may not even be possible. In many cases, primary cells are available because the tissue has been removed or biopsied for diagnostic purposes and can be displaying 809 pathological phenotypes. Primary cells also require specialized culture and media to retain their phenotypes, which can be problematic in linked tissue chip systems, as a common media could

811 prove suboptimal for the different tissues. Finally, adult stem cells grown as organoids (for later 
812 seeding in OoCs) only represent the epithelial component of the tissue, not the stroma or 813 vasculature, limiting their application.

814

815 [bH2] iPSCs: Stem cell-derived sources are a potential solution to cell sourcing difficulties for 816 tissue chips because they are potentially infinitely renewable and can be from either healthy or 817 diseased populations. These iPSCs provide huge potential for populating tissue chips because 818 individuals could have platforms created that model their tissues and disease phenotypes. This 819 also allows creation of isogenic cell lines for genetic disorders, in which the resulting iPSCs can 820 be genetically engineered to either harbor the disease-specific mutation or not, allowing 821 opportunities to study the genetic impact of a disorder with unparalleled specificity.

823 Drawbacks of iPSC-derived tissues include the immature or fetal phenotypes (for example:

824 cardiomyocytes; kidney; and liver) of the cells, which can limit their utility. The time and 825 resources needed for creation and passaging of cell lines, and later differentiation, is long (nine 826 months or more for some neural tissues) and expensive compared to the ease of buying 827 commercially available cells. Also, cells may retain an 'epigenetic memory' of their donor 828 tissues ${ }^{160}$ depending on the number of passages, which can limit directed differentiation for 829 specific tissues. 


\section{$831 \quad$ Figure legends}

\section{Figure 1 |Utility of OoCs in a variety of stages of drug development}

833 Drug development is a dynamic environment for data feedforward and feedback between

834 multiple stages and processes, being described as a 'dynamic map' ${ }^{161}$. These dynamic maps

835 provide a framework for understanding modern drug development and include activities and

836 processes such as Lead Identification, Clinical Research and Development and Regulatory

837 Review. OoCs can be informative in a number of these neighborhoods. On this schematic of an

838 OoC surrounded by the multiple stages and processes of drug development, green components

839 represent the known current or shortly predicted use of OoCs and blue components represent

840 the possible and predicted utility. Many OoCs are currently at the 'Basic science research stage'.

841 Use of OoCs in the 'Medical landscape' stage includes use for precision medicine and patient-

842 specific treatments. 'Clinical research and development' use would include patient subgroup

843 stratification and projects under the NIH "Clinical Trials on a Chip" program, as an example.

844 'Regulatory review' refers to IND and NDA data. 'Post marketing' refers to adverse drug

845 reaction reporting and drug repurposing efforts. References are included for examples of OoC

846 use in these areas. [PR: permissions for need to be included]. 
Figure 2 | Examples of features and platform designs for organs on chips

849 Diverse platform design and key design features for organs on chips allow a broad range of data

850 readouts which can be used for computational modeling as part of the drug discovery process.

851 A broad diversity of tissue platforms highlights key common features - the 3-dimensions for

852 tissue culture, inclusion of multiple cell types, and modeling of biomechanical forces that 853 recreate the in vivo environment.

854 a) Transwell systems allow barrier modeling and fluid flow across a permeable membrane for 855 media exchange and cell-cell interaction. In this example, Caco2 and mucussecreting HT29-MTX intestinal cells create the gut apical side, with immature dendritic cells seeded on the basal side and left to mature, creating a barrier model of the gut. On the

b) Platforms with diamond-shaped cell chambers ( $2 \mathrm{~mm}$ wide by $1 \mathrm{~mm}$ high) allow for seeding with human endothelial colony-forming cell-derived endothelial cells (ECFC-EC, in green) which self-organize into perfusable microvasculature, with cell media supplied via microfluidic channels flowing from bottom to top. Seeding with colorectal cancer cells (HCT116 cells, in blue) forms vascularized microtumors which can be used to screen chemotherapeutics for safety and efficacy. Histology allows clear localization and visualization of cell interactions, such as the vascularization of microtumors and the perfusion of media through the system (rhodamine B dextran, in red). Adapted with permission from ${ }^{29}$.

c) A vascularized liver acinus model (VLAMPS - left) consisting of cells in collagen sandwiched between three glass layers allows 3D layering of multiple liver cell types representing the liver acinus. (Right) Oxygen zonation can be computationally modeled by calculating the rate of media flow in the microfluidic channels, creating 3 distinct zones (oxygen rich; intermediate; and oxygen-poor) on the platform which recreate the liver sinusoid and establish a metabolic gradient similar to that seen in vivo. LECM; liver extracellular matrix. 
876 PET; polyethylene terephthalate. LSECs; liver sinusoidal endothelial cells. Adapted with permission from ${ }^{62}$.

878 [PR: permissions for panels $a, b$, and $c$ need to be included].

879 

target drug effects and create "body-on-a-chip" systems. The modules and media can be

882 linked by (A) pneumatic or electromagnetic pumps, (B and C) peristaltic flow, or (D) media 883 circulated by hydrostatic flow driven by gravity.

884 a) (Left) This female reproductive system MPS contains 5 tissue modules (ovary, cervix, uterus, fallopian tube and liver) and models the hormonal profile of the female menstrual cycle and pregnancy which can be useful for assessing female reproductive toxicity. (Right) The modules are linked by a complex series of internal valves and pumps under the tissue construct inserts and flow of tissue-specific media and hormones are driven by pneumatic pumps powered by electromagnets. Adapted from ${ }^{143}$.

b) (Left) A simplified schematic of a linked multi-organ system for investigating doxorubicin-induced toxicity on liver, heart, bone, and various other tissues e.g. brain. The platform consists of individual tissue constructs cultured in multiple modular 'inserts', set into a platform with the same footprint as a standard 6-well laboratory plate. In this example, 4 tissue types can be replicated in triplicate on a single plate. (Right) Schematic of the side view of the platform. Underneath each tissue insert lies a permeable membrane lined with endothelial cells, perfused by a recirculating vascular medium driven by peristaltic pump. The system allows for optimal cell culture for each tissue type as well as inclusion of common circulating factors such as immune cells, hormones and exosomes. Adapted with permission from ${ }^{162}$.

c) A robotic system with inbuilt microscope, peristaltic pump, and automatic fluid handling named the 'Interrogator' can house up to 10 OoCs for PK/PD and PBMK modeling. Reproduced with permission from ${ }^{128}$.

d) This commercially available multi-organ system from Hesperos Inc. cultures liver, cardiac and skeletal muscle and neurons on a microfluidic chip. Each tissue module is cultured on a plate modified by proprietary surface chemistries to help cells adhere to the surface and act as ECM, and media reservoirs contain a serum-free common medium which is gravity-fed by placing the chip on a laboratory rocker. Cardiac, skeletal and neuronal modules contain microelectrode arrays (MEA) to stimulate and record activity 
in tissue subtypes. Adapted with permission from Schaffer C (November 30 2017) “3D-

910 Bioprinting Conference Showcases Versatility" Genetic Engineering and Biotechnology

$911 \quad$ News magazine Vol 37, No 21. Published by Mary Ann Liebert Inc. publishers.

912 https://www.genengnews.com/magazine/305/3d-bioprinting-conference-showcases-

$913 \quad$ versatility/

914 [PR: permissions for panels $a, b, c$, and $d$ need to be included].

915 
918 extracellular matrix (ECM) - supporting network of macromolecules providing structural and 919 biochemical support to surrounding cells. Promotes cell adhesion and cell-cell communication 920 and produces biochemical cues for tissue growth and maintenance. The ECM is tissue-specific 921 and in animal tissues consists of fibrous elements (collagen, elastin), and links proteins (laminin, 922 fibronectin) and other molecules.

924 hydrogels - highly absorbent and hydrophilic biocompatible 3D polymer networks used to 925 contain cells or drugs for tissue engineering applications. Can consist of natural (collagen, 926 gelatin, agarose) or synthetic components and respond to environmental conditions such as $\mathrm{pH}$. 927 May have both liquid and solid properties. Other uses include wound dressings, contact lenses.

multi-electrode arrays (MEAs) - arrays of 10-1000s of tightly spaced microelectrical sensors 930 designed to record from single cells to networks of cells at sub-millisecond timescales. Can also 931 be used to stimulate cells with precise spatial and temporal characteristics. Used in electrically932 excitable tissues such as cardiac, muscular, neural.

934 pharmacokinetic/pharmacodynamic (PK/PD) modeling - integration of pharmacokinetics (PK 935 movement of drugs through the body) and pharmacodynamics (PD - body's biological response 936 to drugs) into a mathematical model describing dose-concentration-response relationships. Can 937 be used to predict effect and efficacy of drug dosing over time.

940 compartments (predefined organs or tissues) combined with known parameters of

941 concentrations, quantities and transport between compartments used to predict absorption,

942 distribution, metabolism and excretion (ADME) of synthetic or natural chemical substances

943 within the body. 
945 IND (Investigational New Drug) - An application submitted to the US Food and Drug

946 Administration (FDA) to administer novel drug to humans. The first step in the drug review

947 process, which includes information on animal studies, manufacturing protocols, and clinical

948 and personnel protocols. Data gathered becomes part of the New Drug Application (NDA).

949

950 NDA (New Drug Application) - An application submitted to the US FDA requesting permission

951 to sell and market a drug in the US. Information submitted includes data from the IND and is

952 reviewed for safety and efficacy, benefit versus risks, appropriate labelling information, and

953 manufacturing and processing methods.

954 


\section{Related Links}

956 Defense Advanced Research Project Agency (DARPA) funded linked 10 organ system:

957 https://www.darpa.mil/program/microphysiological-systems

959 US National Institutes of Health (NIH), FDA and DARPA funded development of tissue chips to

960 advance regulatory sciences: https://www.nih.gov/news-events/news-releases/nih-fda-

961 announce-collaborative-initiative-fast-track-innovations-public

963 Tissue Chip Testing Centers: https://ncats.nih.gov/tissuechip/projects/centers

964 National Center for Advancing Translational Sciences (NCATS) Tissue Chips in Space:

965 https://ncats.nih.gov/tissuechip/projects/space

967 Good Manufacturing Practice guidelines: https://www.ecfr.gov/cgi-bin/text-

$968 \underline{\text { idx}}$ ?SID=cb7c830642b365274d824a432e118e77\&mc=true\&node=pt21.8.820\&rgn=div5

970 European Union Reference Laboratory for Alternatives to Animal Testing European Centre for

971 the Validation of Alternative (EURL ECVAM): https://ec.europa.eu/jrc/en/eurl/ecvam

973 Organisation for Economic Co-operation and Development (OECD) Guidance Document on the

974 Validation and International Acceptance of New or Updated Test Methods for Hazard

975 Assessment: https://ntp.niehs.nih.gov/iccvam/suppdocs/feddocs/oecd/oecd-gd34.pdf

976

977 Organs on chips - 2017 market overview analysis by Yole Développement:

978 http://www.yole.fr/OrgansOnChips Market.aspx\#.XIP6dVNKiV4

980 The Gartner Hype Cycle for Emerging Technologies 2018:

981 https://www.gartner.com/smarterwithgartner/5-trends-emerge-in-gartner-hype-cycle-for-

982 emerging-technologies-2018/ 
984 IQ Consortium: https://iqconsortium.org/

985

986 human Organ and Disease Model Technologies (hDMT): https://www.hdmt.technology/

987

988 ORCHID: https://h2020-orchid.eu/

989

990 Comprehensive in vitro Proarrhythmia Assay (CiPA): http://cipaproject.org/

991

992 The Center for Advancement of Science in Space (CASIS): https://www.iss-casis.org/

993 


\section{Table 1. Key features of two-dimensional and three-dimensional engineered tissues.}

\begin{tabular}{|l|l|l} 
& & \\
Conventional 2D systems systems & \\
& Organoid & Organ-on-chip
\end{tabular}

\begin{tabular}{|c|c|c|c|}
\hline $\begin{array}{l}\text { Production } \\
\text { characteristics }\end{array}$ & $\begin{array}{l}\text { Grown on rigid flat surfaces, } \\
\text { often as a cellularly } \\
\text { homogeneous monolayer }\end{array}$ & $\begin{array}{l}\text { Embedded in } \\
\text { hydrogels/suspended in 'hanging } \\
\text { drops', and left to self-organize } \\
\text { into multiple cell types }\end{array}$ & $\begin{array}{l}\text { Multiple relevant cell types seeded } \\
\text { into engineered chambers with } \\
\text { perfusion and/or biomechanical } \\
\text { forces included }\end{array}$ \\
\hline $\begin{array}{l}\text { Production } \\
\text { complexity and speed }\end{array}$ & $\begin{array}{l}\text { Generally straightforward } \\
\text { and fast (minutes-days) }\end{array}$ & $\begin{array}{l}\text { Generally straightforward, but } \\
\text { slower (days-weeks) depending } \\
\text { on cell sources }\end{array}$ & $\begin{array}{l}\text { Variable complexity (depends on } \\
\text { platform design), slower (days to } \\
\text { weeks) depending on cell sources } \\
\text { and required tissue maturation } \\
\text { metrics }\end{array}$ \\
\hline $\begin{array}{l}\text { Level of control over } \\
\text { cell architecture }\end{array}$ & High & Very low & High \\
\hline $\begin{array}{l}\text { Maturation of iPSC- } \\
\text { derived cells allowed } \\
\text { by platform* }\end{array}$ & Immature & $\begin{array}{l}\text { Improved but still highly } \\
\text { immature }\end{array}$ & $\begin{array}{l}\text { Platform designs can improve and } \\
\text { encourage cell maturity }{ }^{164}\end{array}$ \\
\hline $\begin{array}{l}\text { Resulting cell } \\
\text { morphology }\end{array}$ & $\begin{array}{l}\text { Unnatural, with limited ECM } \\
\text { composition and contact with } \\
\text { cells }\end{array}$ & $\begin{array}{l}\text { Similar size and shape to in vivo, } \\
\text { allows relevant ECM interaction } \\
\text { during cell proliferation }\end{array}$ & $\begin{array}{l}\text { Similar size and shape to in vivo, } \\
\text { allows relevant ECM interaction } \\
\text { throughout cell lifetime }\end{array}$ \\
\hline $\begin{array}{l}\text { Diffusion of signal } \\
\text { factors and nutrients }\end{array}$ & Short distances possible & $\begin{array}{l}\text { Ineffective transport to interior } \\
\text { can cause cell death or } \\
\text { immaturity }\end{array}$ & $\begin{array}{l}\text { Allows precisely controlled temporal } \\
\text { and spatial gradients }\end{array}$ \\
\hline $\begin{array}{l}\text { Vascularization or } \\
\text { perfusion? }\end{array}$ & $\begin{array}{l}\text { Not possible, generally } \\
\text { perfusion via media change }\end{array}$ & $\begin{array}{l}\text { Depends on cell types but likely } \\
\text { creates non-functional vessels; } \\
\text { externally perfused; can include } \\
\text { fluid flow across tissue surfaces }\end{array}$ & $\begin{array}{l}\text { Yes - by microfluidic channels or } \\
\text { design which can include/create } \\
\text { endothelialized vessels }\end{array}$ \\
\hline $\begin{array}{l}\text { High throughput } \\
\text { feasibility? }\end{array}$ & Yes & $\begin{array}{l}\text { Possibly, depending on } \\
\text { tissue }^{165,166}\end{array}$ & $\begin{array}{l}\text { Depends on platform design; } \\
\text { generally low to medium throughput }\end{array}$ \\
\hline $\begin{array}{l}\text { On-platform assay } \\
\text { and analysis difficulty }\end{array}$ & $\begin{array}{l}\text { Low difficulty, easy access to } \\
\text { cells and readouts }\end{array}$ & $\begin{array}{l}\text { Tissue function analyses possible; } \\
\text { cell separation not possible }\end{array}$ & $\begin{array}{l}\text { Real-time tissue/organ function } \\
\text { analyses possible }\end{array}$ \\
\hline $\begin{array}{l}\text { Variability and in vivo } \\
\text { relevance of resulting } \\
\text { tissues in } \\
\text { manufactured } \\
\text { platform }\end{array}$ & $\begin{array}{l}\text { Low variability and relevance } \\
\text { - simple, homogeneous } \\
\text { cultures }\end{array}$ & $\begin{array}{l}\text { Can be high variability and low } \\
\text { relevance as there is little control } \\
\text { over resulting cell subtypes and } \\
\text { location }\end{array}$ & $\begin{array}{l}\text { Can be low variability and high } \\
\text { relevance - allows high levels of } \\
\text { control over cell type and placement }\end{array}$ \\
\hline
\end{tabular}

$995 *$ *immaturity of iPSC-derived cells still a general issue 
Table 2 - Examples of single tissue OoCs for toxicological assessment

\begin{tabular}{|c|c|c|c|c|}
\hline Tissue/Organ & Platform Characteristics & Challenge & Response & Reference \\
\hline Liver "SQL-SAL" & $\begin{array}{l}\text { Human hepatocytes, } \\
\text { stellate, immune and } \\
\text { endothelial cells are layered } \\
\text { in glass and PDMS } \\
\text { microfluidic chip. } \\
\text { Fluorescent biosensors } \\
\text { included. Survival to } 28 \\
\text { days. }\end{array}$ & $\begin{array}{l}\text { 1. Troglitazone and } \\
\text { nimesulide (hepatotoxic) } \\
\text { 2. Trovafloxacin + LPS and } \\
\text { levofloxacin + LPS (immune- } \\
\text { mediated hepatotoxicity) } \\
\text { 3. Methotrexate (fibrotic } \\
\text { injury) } \\
\text { 4. Caffeine (negative control) }\end{array}$ & $\begin{array}{l}\text { 1. Time and dose-dependent LDH release, } \\
\text { apoptosis, plus decreased albumin and } \\
\text { urea secretion } \\
\text { 2. Increased LDH release and apoptosis with } \\
\text { trovafloxacin + LPS but not levofloxacin + } \\
\text { LPS } \\
\text { 3. Increased fibrotic markers } \\
\text { 4. No effect }\end{array}$ & $\begin{array}{l}\text { Vernetti et al } \\
2016^{61}\end{array}$ \\
\hline Liver & $\begin{array}{l}\text { Primary hepatocytes places } \\
\text { across porous membrane } \\
\text { from LSECs, +/- Kupffer and } \\
\text { stellate cells. Rat, dog, } \\
\text { human species comparisons } \\
\text { possible. }\end{array}$ & $\begin{array}{l}\text { 1. Bosentan (cholestatic) } \\
\text { 2. Acetaminophen } \\
\text { (hepatotoxic) } \\
\text { 3. Methotrexate (fibrotic } \\
\text { injury) }\end{array}$ & $\begin{array}{l}\text { 1. Species-specific albumin decrease; } \\
\text { correlated to clinical response in humans; } \\
\text { bile salt transport inhibition } \\
\text { 2. Glutathione and ATP depletion; } \\
\text { formation of ROS; decreased albumin } \\
\text { secretion } \\
\text { 3. Lipid accumulation (steatosis) and } \\
\text { fibrosis }\end{array}$ & $\begin{array}{l}\text { Jang et al } \\
2019^{63}\end{array}$ \\
\hline Cardiac & $\begin{array}{l}\text { Self-organized iPSC-derived } \\
\text { cardiomyocytes in 3D } \\
\text { microfluidic device }\end{array}$ & $\begin{array}{l}\text { 1. Isoproterenol ( } \beta \text {-adrenergic } \\
\text { agonist) } \\
\text { 2. E-4031 (hERG blocker) } \\
\text { 3. Verapamil (multi-ion } \\
\text { channel blocker) } \\
\text { 4. Metoprolol ( } \beta \text {-adrenergic } \\
\text { antagonist) }\end{array}$ & $\begin{array}{l}\text { Cardiac beat frequencies in line with clinical } \\
\text { data including dose-dependent changes and } \\
\text { arrhythmias concordant with human } \\
\text { cardiotoxicology data }\end{array}$ & $\begin{array}{l}\text { Mathur et al } \\
2015^{64}\end{array}$ \\
\hline Kidney & $\begin{array}{l}\text { Primary human kidney } \\
\text { proximal tubule epithelial } \\
\text { cells seeded to form a } \\
\text { lumen in microfluidic } \\
\text { platform }\end{array}$ & Polymyxin B & $\begin{array}{l}\text { Increased KIM-1 and injury-associated } \\
\text { miRNAs }\end{array}$ & $\begin{array}{l}\text { Weber et al } \\
2018^{78}\end{array}$ \\
\hline
\end{tabular}


10021 Paul, S. M. et al. How to improve R\&D productivity: the pharmaceutical industry's grand 1003 challenge. Nature Reviews Drug Discovery 9, 203, doi:10.1038/nrd3078

1004 https://www.nature.com/articles/nrd3078\#supplementary-information (2010).

10052 Scannell, J. W., Blanckley, A., Boldon, H. \& Warrington, B. Diagnosing the decline in 1006 pharmaceutical R\&D efficiency. Nature Reviews Drug Discovery 11, 191, 1007 doi:10.1038/nrd3681

1008 https://www.nature.com/articles/nrd3681\#supplementary-information (2012).

10093 Seok, J. et al. Genomic responses in mouse models poorly mimic human inflammatory 1010 diseases. Proceedings of the National Academy of Sciences 110, 3507-3512, 1011 doi:10.1073/pnas.1222878110 (2013).

10124 Hay, M., Thomas, D. W., Craighead, J. L., Economides, C. \& Rosenthal, J. Clinical development success rates for investigational drugs. Nature Biotechnology 32, 40, doi:10.1038/nbt.2786

10165 Waring, M. J. et al. An analysis of the attrition of drug candidates from four major pharmaceutical companies. Nature Reviews Drug Discovery 14, 475, doi:10.1038/nrd4609

10206 Sweeney, L. M., Shuler, M. L., Babish, J. G. \& Ghanem, A. A cell culture analogue of rodent physiology: Application to naphthalene toxicology. Toxicology in Vitro 9, 307316, doi:http://dx.doi.org/10.1016/0887-23s33(95)00007-U (1995).

10237 Sin, A. et al. The Design and Fabrication of Three-Chamber Microscale Cell Culture 1024 Analog Devices with Integrated Dissolved Oxygen Sensors. Biotechnology Progress 20, 338-345, doi:10.1021/bp034077d (2004). 1662-1668, doi:10.1126/science.1188302 (2010). 

lung incorporating cyclic biomechanical stretch forces and showing replication of in vivo responses.

10319 Rossi, G., Manfrin, A. \& Lutolf, M. P. Progress and potential in organoid research. Nature Reviews Genetics 19, 671-687, doi:10.1038/s41576-018-0051-9 (2018).

103310 Ingber, D. E. Reverse Engineering Human Pathophysiology with Organs-on-Chips. Cell 1034 164, 1105-1109, doi:https://doi.org/10.1016/j.cell.2016.02.049 (2016).

103511 Pamies, D. et al. A human brain microphysiological system derived from induced 1036 pluripotent stem cells to study neurological diseases and toxicity. ALTEX, 1037 doi:10.14573/altex.1609122 (2016).

103812 Plummer, S. et al. A Human iPSC-derived 3D platform using primary brain cancer cells to 1039 study drug development and personalized medicine. Scientific Reports 9, 1407, 1040 doi:10.1038/s41598-018-38130-0 (2019).

104113 Schwartz, M. P. et al. Human pluripotent stem cell-derived neural constructs for 1042 1043 1044

14 Rothbauer, M., Rosser, J. M., Zirath, H. \& Ertl, P. Tomorrow today: organ-on-a-chip 1045 1046

15 Kasendra, M. et al. Development of a primary human Small Intestine-on-a-Chip using biopsy-derived organoids. Scientific Reports 8, 2871, doi:10.1038/s41598-018-21201-7

105116 Ramme, A. P. et al. Towards an autologous iPSC-derived patient-on-a-chip. bioRxiv, 1052

105317 Vatine, G. D. et al. Human iPSC-Derived Blood-Brain Barrier Chips Enable Disease 1054 1055 Modeling and Personalized Medicine Applications. Cell Stem Cell 24, 995-1005.e1006, doi:https://doi.org/10.1016/i.stem.2019.05.011 (2019). 
105618 Caliari, S. R. \& Burdick, J. A. A practical guide to hydrogels for cell culture. Nature

$1057 \quad$ Methods 13, 405, doi:10.1038/nmeth.3839

1058 https://www.nature.com/articles/nmeth.3839\#supplementary-information (2016).

105919 Crapo, P. M., Tottey, S., Slivka, P. F. \& Badylak, S. F. Effects of Biologic Scaffolds on

$1060 \quad$ Human Stem Cells and Implications for CNS Tissue Engineering. Tissue Engineering Part

$1061 \quad$ A 20, 313-323, doi:10.1089/ten.tea.2013.0186 (2013).

106220 Safaee, H. et al. Tethered Jagged-1 Synergizes with Culture Substrate Stiffness to

1063 Modulate Notch-Induced Myogenic Progenitor Differentiation. Cellular and Molecular

1064 Bioengineering 10, 501-513, doi:10.1007/s12195-017-0506-7 (2017).

106521 Trappmann, B. et al. Matrix degradability controls multicellularity of 3D cell migration.

$1066 \quad$ Nature Communications 8, 371, doi:10.1038/s41467-017-00418-6 (2017).

106722 Shin, D. S. et al. Synthesis of microgel sensors for spatial and temporal monitoring of

1068 protease activity. ACS biomaterials science \& engineering 4, 378-387,

1069 doi:10.1021/acsbiomaterials.7b00017 (2018).

107023 Wikswo, J. P. et al. Engineering Challenges for Instrumenting and Controlling Integrated

1071 Organ-on-Chip Systems. IEEE Transactions on Biomedical Engineering 60, 682-690,

1072 doi:10.1109/TBME.2013.2244891 (2013).

107324 Wikswo, J. P. et al. Scaling and systems biology for integrating multiple organs-on-a-

1074

107525 Johnson, B. P. et al. Hepatocyte circadian clock controls acetaminophen bioactivation

1076

1077

107826

through NADPH-cytochrome P450 oxidoreductase. Proceedings of the National

1079

1080

27 Cyr, K. J., Avaldi, O. M. \& Wikswo, J. P. Circadian hormone control in a human-on-a-chip:

1081

1082 Academy of Sciences 111, 18757, doi:10.1073/pnas.1421708111 (2014).

26 Bass, J. \& Takahashi, J. S. Circadian Integration of Metabolism and Energetics. Science 330, 1349, doi:10.1126/science.1195027 (2010).

108328 Chang, S.-Y. et al. Human liver-kidney model elucidates the mechanisms of aristolochic 1084 acid nephrotoxicity. JCI Insight 2, doi:10.1172/jci.insight.95978 (2017). 
Physically coupled liver and kidney OoCs used to uncover the mechanism of nephrotoxicity of aristolochic acid via bioactivation in the liver, showing utility of coupled OoCs for understanding toxicities.

1088

Phan, D. T. T. et al. A vascularized and perfused organ-on-a-chip platform for large-scale drug screening applications. Lab on a Chip 17, 511-520, doi:10.1039/C6LC01422D (2017).

30 Zhang, C., Zhao, Z., Abdul Rahim, N. A., van Noort, D. \& Yu, H. Towards a human-on-chip: 1093

31 Materne, E.-M. et al. The Multi-organ Chip - A Microfluidic Platform for Long-term 1095

32 Maschmeyer, I. et al. A four-organ-chip for interconnected long-term co-culture of human intestine, liver, skin and kidney equivalents. Lab on a Chip 15, 2688-2699, doi:10.1039/C5LC00392J (2015).

Development of a multi-organ integrated OoC with pulsatile flow which reliably supports homeostasis over 28 days and allows ADME profiling and repeated dose drug

110233 Tsamandouras, N. et al. Integrated Gut and Liver Microphysiological Systems for 1103

34 Oleaga, C. et al. Multi-Organ toxicity demonstration in a functional human in vitro system composed of four organs. Scientific Reports 6, 20030, doi:10.1038/srep20030 Quantitative In Vitro Pharmacokinetic Studies. The AAPS Journal 19, 1499-1512,

\section{https://www.nature.com/articles/srep20030\#supplementary-information (2016).}

Development of a multi-organ pumpless OoC with common medium under serum-free conditions, maintaining tissues to 14 days and profiling accurate acute responses to therapeutic compounds.

35 Oleaga, C. et al. Human-on-a-Chip Systems: Long-Term Electrical and Mechanical Function Monitoring of a Human-on-a-Chip System (Adv. Funct. Mater. 8/2019). Advanced Functional Materials 29, 1970049, doi:10.1002/adfm.201970049 (2019). 
111436 Stone, H. A., Stroock, A. D. \& Ajdari, A. Engineering Flows in Small Devices: Microfluidics

1115 Toward a Lab-on-a-Chip. Annual Review of Fluid Mechanics 36, 381-411,

1116 doi:10.1146/annurev.fluid.36.050802.122124 (2004).

111737 Lochovsky, C., Yasotharan, S. \& Günther, A. Bubbles no more: in-plane trapping and

1118 removal of bubbles in microfluidic devices. Lab on a Chip 12, 595-601,

1119 doi:10.1039/C1LC20817A (2012).

112038 Kaarj, K. \& Yoon, J.-Y. Methods of Delivering Mechanical Stimuli to Organ-on-a-Chip.

$1121 \quad$ Micromachines 10, doi:10.3390/mi10100700 (2019).

112239 Kim, H. J., Huh, D., Hamilton, G. \& Ingber, D. E. Human gut-on-a-chip inhabited by

1123 microbial flora that experiences intestinal peristalsis-like motions and flow. Lab on $a$

1124 Chip 12, 2165-2174, doi:10.1039/C2LC40074J (2012).

112540 Maoz, B. M. et al. Organs-on-Chips with combined multi-electrode array and

1126 transepithelial electrical resistance measurement capabilities. Lab on a Chip 17, 2294-

1127 2302, doi:10.1039/C7LC00412E (2017).

112841 Herland, A. et al. Distinct Contributions of Astrocytes and Pericytes to

$1129 \quad$ Neuroinflammation Identified in a 3D Human Blood-Brain Barrier on a Chip. PLOS ONE

1130 11, e0150360, doi:10.1371/journal.pone.0150360 (2016).

113142 Musah, S. et al. Mature induced-pluripotent-stem-cell-derived human podocytes

1132 reconstitute kidney glomerular-capillary-wall function on a chip. Nature biomedical

1133 engineering 1, 0069, doi:10.1038/s41551-017-0069 (2017).

113443 Agarwal, A., Goss, J. A., Cho, A., McCain, M. L. \& Parker, K. K. Microfluidic heart on a chip

1135 for higher throughput pharmacological studies. Lab on a chip 13, 3599-3608,

1136 doi:10.1039/c3lc50350j (2013).

$113744 \quad$ Nunes, S. S. et al. Biowire: a platform for maturation of human pluripotent stem cell-

1138 derived cardiomyocytes. Nature Methods 10, 781, doi:10.1038/nmeth.2524

1139 https://www.nature.com/articles/nmeth.2524\#supplementary-information (2013).

114045 Lee-Montiel, F. T. et al. Control of oxygen tension recapitulates zone-specific functions

1141 in human liver microphysiology systems. Experimental Biology and Medicine 242, 1617-

$1142 \quad$ 1632, doi:10.1177/1535370217703978 (2017). 
114346 Senutovitch, N. et al. Fluorescent protein biosensors applied to microphysiological

$1144 \quad$ systems. Experimental Biology and Medicine 240, 795-808,

1145 doi:10.1177/1535370215584934 (2015).

114647 Zhang, Y. S. et al. Multisensor-integrated organs-on-chips platform for automated and

1147 continual in situ monitoring of organoid behaviors. Proceedings of the National

$1148 \quad$ Academy of Sciences 114, E2293 (2017).

1149 Advanced multi-organ platform for automated control and biosensing over multiple 1150 days, including $\mathrm{pH}, \mathbf{0 2}$, temperature, protein biomarker presence and microscopes for 1151 imaging. Demonstrated cardio- and hepatotoxicity from chronic and acute drug 1152 dosing.

115348 Toepke, M. W. \& Beebe, D. J. PDMS absorption of small molecules and consequences in 1154 microfluidic applications. Lab on a Chip 6, 1484-1486, doi:10.1039/B612140C (2006).

115549 Markov, D. A., Lillie, E. M., Garbett, S. P. \& McCawley, L. J. Variation in diffusion of gases 1156 through PDMS due to plasma surface treatment and storage conditions. Biomedical 1157 microdevices 16, 91-96, doi:10.1007/s10544-013-9808-2 (2014).

115850 Tan, S. H., Nguyen, N.-T., Chua, Y. C. \& Kang, T. G. Oxygen plasma treatment for reducing 1159 hydrophobicity of a sealed polydimethylsiloxane microchannel. Biomicrofluidics 4, $1160 \quad$ 032204, doi:10.1063/1.3466882 (2010).

$116151 \quad$ Chuah, Y. J. et al. Simple surface engineering of polydimethylsiloxane with 1162 polydopamine for stabilized mesenchymal stem cell adhesion and multipotency.

1163 Scientific Reports 5, 18162, doi:10.1038/srep18162

1164 https://www.nature.com/articles/srep18162\#supplementary-information (2015).

116552 van Meer, B. J. et al. Small molecule absorption by PDMS in the context of drug 1166 response bioassays. Biochemical and Biophysical Research Communications 482, 3231167 328, doi:https://doi.org/10.1016/j.bbrc.2016.11.062 (2017).

116853 Regehr, K. J. et al. Biological implications of polydimethylsiloxane-based microfluidic cell 1169 culture. Lab on a chip 9, 2132-2139, doi:10.1039/b903043c (2009). 
117054 Suntharalingam, G. et al. Cytokine Storm in a Phase 1 Trial of the Anti-CD28 Monoclonal

1171 Antibody TGN1412. New England Journal of Medicine 355, 1018-1028,

1172 doi:10.1056/NEJMoa063842 (2006).

117355 Kaur, R., Sidhu, P. \& Singh, S. What failed BIA 10-2474 Phase I clinical trial? Global

1174 speculations and recommendations for future Phase I trials. Journal of pharmacology \&

1175 pharmacotherapeutics 7, 120-126, doi:10.4103/0976-500X.189661 (2016).

$117656 \quad$ Fowler, S. et al. Microphysiological systems for ADME-related applications: current

1177 status and recommendations for system development and characterization. Lab on $a$

1178 Chip 20, 446-467, doi:10.1039/C9LC00857H (2020).

117957 Fabre, K. et al. Introduction to a manuscript series on the characterization and use of 1180 microphysiological systems (MPS) in pharmaceutical safety and ADME applications. Lab 1181 on a Chip 20, 1049-1057, doi:10.1039/C9LC01168D (2020).

118258 Rudmann, D. G. The Emergence of Microphysiological Systems (Organs-on-chips) as 1183 Paradigm-changing Tools for Toxicologic Pathology. Toxicologic Pathology 47, 4-10, 1184 doi:10.1177/0192623318809065 (2018).

118559 Gerets, H. H. J. et al. Characterization of primary human hepatocytes, HepG2 cells, and 1186 HepaRG cells at the mRNA level and CYP activity in response to inducers and their 1187 predictivity for the detection of human hepatotoxins. Cell biology and toxicology 28, 691188 87, doi:10.1007/s10565-011-9208-4 (2012).

118960 Heslop, J. A. et al. Mechanistic evaluation of primary human hepatocyte culture using 1190 global proteomic analysis reveals a selective dedifferentiation profile. Archives of 1191 Toxicology 91, 439-452, doi:10.1007/s00204-016-1694-y (2017).

119261 Vernetti, L. A. et al. A human liver microphysiology platform for investigating physiology, 1193 drug safety, and disease models. Experimental Biology and Medicine 241, 101-114, 1194 doi:10.1177/1535370215592121 (2016).

119562 Li, X., George, S. M., Vernetti, L., Gough, A. H. \& Taylor, D. L. A glass-based, continuously 1196 zonated and vascularized human liver acinus microphysiological system (vLAMPS) 1197 designed for experimental modeling of diseases and ADME/TOX. Lab on a Chip 18, 26141198 2631, doi:10.1039/C8LC00418H (2018). 
119963 Jang, K.-J. et al. Reproducing human and cross-species drug toxicities using a Liver-Chip.

$1200 \quad$ Science Translational Medicine 11, eaax5516, doi:10.1126/scitranslmed.aax5516 (2019).

1201 First paper to compare rat, dog and human microfluidic multi-cellular liver chips after exposure to hepatotoxic compounds. A fibrotic liver model showed species differences highlighting species-specific differences in drug metabolism and toxicity.

64 Mathur, A. et al. Human iPSC-based Cardiac Microphysiological System For Drug

Screening Applications. Scientific Reports 5, 8883, doi:10.1038/srep08883

http://www.nature.com/articles/srep08883\#supplementary-information (2015).

120765 Ahn, S. et al. Mussel-inspired 3D fiber scaffolds for heart-on-a-chip toxicity studies of 1208 engineered nanomaterials. Analytical and Bioanalytical Chemistry 410, 6141-6154, 1209 doi:10.1007/s00216-018-1106-7 (2018).

121066 Marsano, A. et al. Beating heart on a chip: a novel microfluidic platform to generate functional 3D cardiac microtissues. Lab on a Chip 16, 599-610, doi:10.1039/C5LC01356A

121367 Lind, J. U. et al. Instrumented cardiac microphysiological devices via multimaterial three1214 dimensional printing. Nature Materials 16, 303, doi:10.1038/nmat4782

https://www.nature.com/articles/nmat4782\#supplementary-information (2016).

121668 Capulli, A. K., MacQueen, L. A., O'Connor, B. B., Dauth, S. \& Parker, K. K. Acute pergolide exposure stiffens engineered valve interstitial cell tissues and reduces contractility in vitro. Cardiovascular Pathology 25, 316-324, doi:http://dx.doi.org/10.1016/j.carpath.2016.04.004 (2016).

122470 Sheehy, S. P. et al. Toward improved myocardial maturity in an organ-on-chip platform with immature cardiac myocytes. Experimental Biology and Medicine 242, 1643-1656, doi:10.1177/1535370217701006 (2017). 
122771 Ronaldson-Bouchard, K. et al. Advanced maturation of human cardiac tissue grown from 1228 pluripotent stem cells. Nature 556, 239-243, doi:10.1038/s41586-018-0016-3 (2018). OoC used to show enhanced maturation of stem cell-derived cardiac tissues in 3D when subject to electrical stimulation protocols, addressing a key challenge in cardiac stem cell differentiation.

123272 Zhao, Y. et al. A Platform for Generation of Chamber-Specific Cardiac Tissues and 1233 Disease Modeling. Cell 176, 913-927.e918, doi:https://doi.org/10.1016/i.cell.2018.11.042 (2019).

123573 Mills, R. J. et al. Functional screening in human cardiac organoids reveals a metabolic 1236 mechanism for cardiomyocyte cell cycle arrest. Proceedings of the National Academy of 1237 Sciences 114, E8372, doi:10.1073/pnas.1707316114 (2017).

123874 Giacomelli, E. et al. Human-iPSC-Derived Cardiac Stromal Cells Enhance Maturation in 1239 3D Cardiac Microtissues and Reveal Non-cardiomyocyte Contributions to Heart Disease.

124175 Jang, K.-J. et al. Human kidney proximal tubule-on-a-chip for drug transport and 1242 nephrotoxicity assessment. Integrative Biology 5, 1119-1129, doi:10.1039/C3IB40049B 1243 (2013).

124476 Kim, S. et al. Pharmacokinetic profile that reduces nephrotoxicity of gentamicin in a 1245 perfused kidney-on-a-chip. Biofabrication 8, 015021, doi:10.1088/1758-

1248 1249

125078 Weber, E. J. et al. Human kidney on a chip assessment of polymyxin antibiotic 1251 nephrotoxicity. JCI Insight 3, doi:10.1172/jci.insight.123673 (2018).

125279 Kim, H. J. \& Ingber, D. E. Gut-on-a-Chip microenvironment induces human intestinal cells 1253 to undergo villus differentiation. Integrative Biology 5, 1130-1140, 1254 doi:10.1039/C3IB40126J (2013). 
$125580 \quad$ Workman, M. J. et al. Engineered human pluripotent-stem-cell-derived intestinal tissues 1256 with a functional enteric nervous system. Nature Medicine 23, 49, doi:10.1038/nm.4233

1257 https://www.nature.com/articles/nm.4233\#supplementary-information (2016).

125881 Williamson, I. A. et al. A High-Throughput Organoid Microinjection Platform to Study

1259 Gastrointestinal Microbiota and Luminal Physiology. Cellular and Molecular

1260 Gastroenterology and Hepatology 6, 301-319,

1261 doi:https://doi.org/10.1016/j.jemgh.2018.05.004 (2018).

126282 Shah, P. et al. A microfluidics-based in vitro model of the gastrointestinal human-

1263 microbe interface. Nature Communications 7, 11535, doi:10.1038/ncomms11535

1264 https://www.nature.com/articles/ncomms11535\#supplementary-information (2016).

$126583 \quad$ Workman, M. J. et al. Enhanced Utilization of Induced Pluripotent Stem Cell-Derived $1266 \quad$ Human Intestinal Organoids Using Microengineered Chips. Cellular and Molecular

1267 Gastroenterology and Hepatology 5, 669-677.e662,

1268 doi:https://doi.org/10.1016/j.jcmgh.2017.12.008 (2018).

126984 Tovaglieri, A. et al. Species-specific enhancement of enterohemorrhagic E. coli

1270 pathogenesis mediated by microbiome metabolites. Microbiome 7, 43,

1271 doi:10.1186/s40168-019-0650-5 (2019).

127285 Jalili-Firoozinezhad, S. et al. A complex human gut microbiome cultured in an anaerobic

1273

1274

1275

86 Benam, K. H. et al. in 3D Cell Culture: Methods and Protocols (ed Zuzana Koledova) 345-

1276365 (Springer New York, 2017).

127787 Benam, K. H. et al. Matched-Comparative Modeling of Normal and Diseased Human

$1278 \quad$ Airway Responses Using a Microengineered Breathing Lung Chip. Cell Systems 3, 456-

1279 466.e454, doi:10.1016/j.cels.2016.10.003 (2016).

$128088 \quad$ Blundell, C. et al. Placental Drug Transport-on-a-Chip: A Microengineered In Vitro Model 1281 of Transporter-Mediated Drug Efflux in the Human Placental Barrier. Advanced $1282 \quad$ Healthcare Materials 7, 1700786, doi:10.1002/adhm.201700786 (2018). 
128389 Yin, F. et al. A 3D human placenta-on-a-chip model to probe nanoparticle exposure at 1284 the placental barrier. Toxicology in Vitro 54, 105-113,

1285 doi:https://doi.org/10.1016/j.tiv.2018.08.014 (2019).

128690 Sobrino, A. et al. 3D microtumors in vitro supported by perfused vascular networks.

1287 Scientific Reports 6, 31589, doi:10.1038/srep31589 (2016).

$128891 \quad$ Barrile, R. et al. Organ-on-Chip Recapitulates Thrombosis Induced by an anti-CD154

1289 Monoclonal Antibody: Translational Potential of Advanced Microengineered Systems.

1290 Clinical Pharmacology \& Therapeutics 104, 1240-1248, doi:10.1002/cpt.1054 (2018).

129192 Cook, D. et al. Lessons learned from the fate of AstraZeneca's drug pipeline: a five-

1292 dimensional framework. Nat Rev Drug Discov 13, 419-431, doi:10.1038/nrd4309 (2014).

129393 Horton, R. E. et al. Angiotensin II Induced Cardiac Dysfunction on a Chip. PLOS ONE 11, $1294 \quad$ e0146415, doi:10.1371/journal.pone.0146415 (2016).

129594 Hinson, J. T. et al. Titin mutations in iPS cells define sarcomere insufficiency as a cause of 1296 dilated cardiomyopathy. Science 349, 982-986, doi:10.1126/science.aaa5458 (2015).

129795 Nesmith, A. P., Agarwal, A., McCain, M. L. \& Parker, K. K. Human airway musculature on 1298 a chip: an in vitro model of allergic asthmatic bronchoconstriction and bronchodilation. Lab on a Chip 14, 3925-3936, doi:10.1039/C4LC00688G (2014).

1301

1302

1303

1304

97 Cruz, N. M. et al. Organoid cystogenesis reveals a critical role of microenvironment in 1305 human polycystic kidney disease. Nature Materials 16, 1112, doi:10.1038/nmat4994

1306 https://www.nature.com/articles/nmat4994\#supplementary-information (2017).

$130798 \quad$ Faal, T. et al. Induction of Mesoderm and Neural Crest-Derived Pericytes from Human 1308 Pluripotent Stem Cells to Study Blood-Brain Barrier Interactions. Stem Cell Reports 12, 1309 451-460, doi:https://doi.org/10.1016/j.stemcr.2019.01.005 (2019).

99 Rooney, G. E. et al. Human iPS Cell-Derived Neurons Uncover the Impact of Increased Ras Signaling in Costello Syndrome. The Journal of Neuroscience 36, 142 (2016). 
1312100 Atchison, L., Zhang, H., Cao, K. \& Truskey, G. A. A Tissue Engineered Blood Vessel Model 1313 of Hutchinson-Gilford Progeria Syndrome Using Human iPSC-derived Smooth Muscle 1314 Cells. Scientific Reports 7, 8168, doi:10.1038/s41598-017-08632-4 (2017).

1315101 Wang, G. et al. Modeling the mitochondrial cardiomyopathy of Barth syndrome with 1316 induced pluripotent stem cell and heart-on-chip technologies. Nat Med 20, 616-623, 1317 doi:10.1038/nm.3545

1318 http://www.nature.com/nm/journal/v20/n6/abs/nm.3545.html\#supplementary-information 1319 (2014).

Rare pediatric disease modeled on OoC and mechanism of pathology uncovered using gene editing techniques.

102 Ben Jehuda, R., Shemer, Y. \& Binah, O. Genome Editing in Induced Pluripotent Stem Cells using CRISPR/Cas9. Stem Cell Reviews and Reports 14, 323-336,

103 Nguyen, D.-H. T. et al. Biomimetic model to reconstitute angiogenic sprouting

104 Montanez-Sauri, S. I., Sung, K. E., Berthier, E. \& Beebe, D. J. Enabling screening in 3D

105 Zervantonakis, I. K. et al. Three-dimensional microfluidic model for tumor cell intravasation and endothelial barrier function. Proceedings of the National Academy of

106 Jeon, J. S. et al. Human 3D vascularized organotypic microfluidic assays to study breast 
1341108 Shirure, V. S. et al. Tumor-on-a-chip platform to investigate progression and drug 1342 sensitivity in cell lines and patient-derived organoids. Lab on a Chip 18, 3687-3702, 1343 doi:10.1039/C8LC00596F (2018).

1344109 Regier, M. C. et al. Transitions from mono- to co- to tri-culture uniquely affect gene 1345 expression in breast cancer, stromal, and immune compartments. Biomedical Microdevices 18, 70, doi:10.1007/s10544-016-0083-x (2016).

1347110 Marturano-Kruik, A. et al. Human bone perivascular niche-on-a-chip for studying metastatic colonization. Proceedings of the National Academy of Sciences 115, 1256,

111 Kim, S., Lee, H., Chung, M. \& Jeon, N. L. Engineering of functional, perfusable 3D microvascular networks on a chip. Lab on a Chip 13, 1489-1500, doi:10.1039/C3LC41320A (2013).

112 Miller, C. P., Tsuchida, C., Zheng, Y., Himmelfarb, J. \& Akilesh, S. A 3D Human Renal Cell Carcinoma-on-a-Chip for the Study of Tumor Angiogenesis. Neoplasia 20, 610-620, doi:https://doi.org/10.1016/j.neo.2018.02.011 (2018).

113 Hassell, B. A. et al. Human Organ Chip Models Recapitulate Orthotopic Lung Cancer Growth, Therapeutic Responses, and Tumor Dormancy In Vitro. Cell Reports 21, 508516, doi:https://doi.org/10.1016/j.celrep.2017.09.043 (2017).

114 Lee, J.-H. et al. Microfluidic co-culture of pancreatic tumor spheroids with stellate cells

115 Jeong, S.-Y., Lee, J.-H., Shin, Y., Chung, S. \& Kuh, H.-J. Co-Culture of Tumor Spheroids and

116 Rizvi, l. et al. Flow induces epithelial-mesenchymal transition, cellular heterogeneity and biomarker modulation in 3D ovarian cancer nodules. Proceedings of the National Academy of Sciences 110, E1974, doi:10.1073/pnas.1216989110 (2013). 
1370117 Li, R. et al. Macrophage-Secreted TNF $\alpha$ and TGF $\beta 1$ Influence Migration Speed and 1371 Persistence of Cancer Cells in 3D Tissue Culture via Independent Pathways. Cancer 1372 Research 77, 279, doi:10.1158/0008-5472.CAN-16-0442 (2017).

1373118 Wang, N. et al. 3D microfluidic in vitro model and bioinformatics integration to study the effects of Spatholobi Caulis tannin in cervical cancer. Scientific Reports 8, 12285, doi:10.1038/s41598-018-29848-y (2018).

119 Low, L. A. \& Tagle, D. A. Tissue chips to aid drug development and modeling for rare diseases. Expert Opin Orphan D 4, 1113-1121, doi:10.1080/21678707.2016.1244479 (2016).

120 Shik Mun, K. et al. Patient-derived pancreas-on-a-chip to model cystic fibrosis-related disorders. Nature Communications 10, 3124, doi:10.1038/s41467-019-11178-w (2019).

122 Takebe, T., Zhang, B. \& Radisic, M. Synergistic Engineering: Organoids Meet Organs-ona-Chip. Cell Stem Cell 21, 297-300, doi:https://doi.org/10.1016/j.stem.2017.08.016 (2017).

123 Park, S. E., Georgescu, A. \& Huh, D. Organoids-on-a-chip. Science 364, 960, doi:10.1126/science.aaw7894 (2019).

125 Zhang, Y. S. et al. Bioprinting 3D microfibrous scaffolds for engineering endothelialized myocardium and heart-on-a-chip. Biomaterials 110, 45-59, doi:https://doi.org/10.1016/i.biomaterials.2016.09.003 (2016).

124 Takebe, T. et al. Vascularized and Complex Organ Buds from Diverse Tissues via Mesenchymal Cell-Driven Condensation. Cell Stem Cell 16, 556-565, doi:https://doi.org/10.1016/i.stem.2015.03.004 (2015).

126 Park, D., Lee, J., Chung, J. J., Jung, Y. \& Kim, S. H. Integrating Organs-on-Chips: Multiplexing, Scaling, Vascularization, and Innervation. Trends in Biotechnology, doi:https://doi.org/10.1016/j.tibtech.2019.06.006 (2019). 
127 Edington, C. D. et al. Interconnected Microphysiological Systems for Quantitative Biology and Pharmacology Studies. Scientific Reports 8, 4530, doi:10.1038/s41598-01822749-0 (2018).

Multi-organ microfluidic "physiome-on-a-chip" platform modeling up to 10 organs for 4 weeks with pharmacokinetic analysis of diclofenac metabolism, noting general design and operational principles for multi-organ platforms.

128 Novak, R. et al. Robotic fluidic coupling and interrogation of multiple vascularized organ chips. Nature Biomedical Engineering 4, 407-420, doi:10.1038/s41551-019-0497-x (2020).

Multi-organ linked system for up to 10 OoC for 3 weeks in automated culture and perfusion machine capable of medium addition, fluidic linking, sample collection and in situ microscopy.

Shim, M. K. et al. Carrier-free nanoparticles of cathepsin B-cleavable peptide-conjugated doxorubicin prodrug for cancer targeting therapy. Journal of Controlled Release 294,

133 Satoh, T. et al. A multi-throughput multi-organ-on-a-chip system on a plate formatted pneumatic pressure-driven medium circulation platform. Lab on a Chip 18, 115-125,

1423134 Boos, J. A., Misun, P. M., Michlmayr, A., Hierlemann, A. \& Frey, O. Microfluidic 376-389, doi:https://doi.org/10.1016/j.jconrel.2018.11.032 (2019).

Al-Malahmeh, A. J. et al. Physiologically based kinetic modeling of the bioactivation of myristicin. Archives of Toxicology 91, 713-734, doi:10.1007/s00204-016-1752-5 (2017).

Schurdak, M. E. et al. in Phenotypic Screening: Methods and Protocols (ed Bridget Wagner) 207-222 (Springer New York, 2018).

Oliver, C. R. et al. A platform for artificial intelligence based identification of the extravasation potential of cancer cells into the brain metastatic niche. Lab on a Chip, doi:10.1039/C8LC01387J (2019). doi:10.1039/C7LC00952F (2018). Multitissue Platform for Advanced Embryotoxicity Testing In Vitro. Adv Sci (Weinh) 6, 1900294-1900294, doi:10.1002/advs.201900294 (2019). 
1426135 Vunjak-Novakovic, G., Bhatia, S., Chen, C. \& Hirschi, K. HeLiVa platform: integrated 1427 heart-liver-vascular systems for drug testing in human health and disease. Stem Cell 1428 Research \& Therapy 4, 1-6, doi:10.1186/scrt369 (2013).

1429136 Vernetti, L. et al. Functional Coupling of Human Microphysiology Systems: Intestine, 1430 Liver, Kidney Proximal Tubule, Blood-Brain Barrier and Skeletal Muscle. Scientific Reports $1431 \quad 7,42296$, doi:10.1038/srep42296

1432 http://www.nature.com/articles/srep42296\#supplementary-information (2017).

1433137 Brown, J. A. et al. Recreating blood-brain barrier physiology and structure on chip: A 1434 novel neurovascular microfluidic bioreactor. Biomicrofluidics 9, 054124, 1435 doi:10.1063/1.4934713 (2015).

1436138 Brown, J. A. et al. Metabolic consequences of inflammatory disruption of the blood1437 brain barrier in an organ-on-chip model of the human neurovascular unit. Journal of 1438 Neuroinflammation 13, 306, doi:10.1186/s12974-016-0760-y (2016).

1439139 Maoz, B. M. et al. A linked organ-on-chip model of the human neurovascular unit 1440 reveals the metabolic coupling of endothelial and neuronal cells. Nature Biotechnology $1441 \quad 36,865$, doi:10.1038/nbt.4226

1442 https://www.nature.com/articles/nbt.4226\#supplementary-information (2018).

1443140 Chen, W. L. K. et al. Integrated gut/liver microphysiological systems elucidates 1444 inflammatory inter-tissue crosstalk. Biotechnology and Bioengineering 114, 2648-2659, 1445 doi:10.1002/bit.26370 (2017).

1446141 Esch, M. B., Ueno, H., Applegate, D. R. \& Shuler, M. L. Modular, pumpless body-on-a1447 chip platform for the co-culture of GI tract epithelium and 3D primary liver tissue. Lab 1448 on a Chip 16, 2719-2729, doi:10.1039/C6LC00461J (2016).

1449142 Loskill, P., Marcus, S. G., Mathur, A., Reese, W. M. \& Healy, K. E. $\mu$ Organo: A Lego ${ }^{\circledR}$-Like $1450 \quad$ Plug \& Play System for Modular Multi-Organ-Chips. PLOS ONE 10, e0139587, 1451 doi:10.1371/journal.pone.0139587 (2015).

1452143 Xiao, S. et al. A microfluidic culture model of the human reproductive tract and 28-day 1453 menstrual cycle. Nature Communications 8, 14584, doi:10.1038/ncomms14584

1454 http://www.nature.com/articles/ncomms14584\#supplementary-information (2017). 
Human female reproductive system and cycle recreated on 5-organ platform with inclusion of endocrine signaling to mimic hormonal markers of pregnancy as a tool for female reproductive toxicity assessment.

1458144 Skardal, A., Shupe, T. \& Atala, A. Organoid-on-a-chip and body-on-a-chip systems for drug screening and disease modeling. Drug Discovery Today 21, 1399-1411, doi:https://doi.org/10.1016/j.drudis.2016.07.003 (2016).

145 Skardal, A. et al. Multi-tissue interactions in an integrated three-tissue organ-on-a-chip platform. Scientific Reports 7, 8837, doi:10.1038/s41598-017-08879-x (2017).

146 Oleaga, C. et al. Investigation of the effect of hepatic metabolism on off-target cardiotoxicity in a multi-organ human-on-a-chip system. Biomaterials 182, 176-190, doi:https://doi.org/10.1016/j.biomaterials.2018.07.062 (2018).

148 Roberts, R. A. et al. Reducing attrition in drug development: smart loading preclinical safety assessment. Drug Discovery Today 19, 341-347, doi:https://doi.org/10.1016/i.drudis.2013.11.014 (2014). Journal 14, 207-210, doi:http://dx.doi.org/10.1016/j.csbj.2016.04.003 (2016).

151 Lee, J. et al. Recent advances in genome editing of stem cells for drug discovery and therapeutic application. Pharmacology \& Therapeutics, 107501, doi:https://doi.org/10.1016/j.pharmthera.2020.107501 (2020). 
1482152 Franzen, N. et al. Impact of organ-on-a-chip technology on pharmaceutical R\&D costs. Drug Discovery Today 24, 1720-1724, doi:https://doi.org/10.1016/j.drudis.2019.06.003 (2019).

153 Peterson, N. C., Mahalingaiah, P. K., Fullerton, A. \& Di Piazza, M. Application of microphysiological systems in biopharmaceutical research and development. Lab on a Chip 20, 697-708, doi:10.1039/C9LC00962K (2020).

154 Hardwick, R. N. et al. Drug-induced skin toxicity: gaps in preclinical testing cascade as opportunities for complex in vitro models and assays. Lab on a Chip 20, 199-214, doi:10.1039/C9LC00519F (2020).

Ainslie, G. R. et al. Microphysiological lung models to evaluate the safety of new pharmaceutical modalities: a biopharmaceutical perspective. Lab on a Chip 19, 31523161, doi:10.1039/C9LC00492K (2019).

157 Phillips, J. A. et al. A pharmaceutical industry perspective on microphysiological kidney 1498 systems for evaluation of safety for new therapies. Lab on a Chip 20,468-476, doi:10.1039/C9LC00925F (2020). assessment: potential for microphysiological systems. Lab on a Chip 20, 1177-1190, doi:10.1039/C9LC01107B (2020).

161 Wagner, J. A. et al. Application of a Dynamic Map for Learning, Communicating, 1508 1509 Navigating, and Improving Therapeutic Development. Clinical and Translational Science 11, 166-174, doi:10.1111/cts.12531 (2017). 
1510162 Ronaldson-Bouchard, K. \& Vunjak-Novakovic, G. Organs-on-a-Chip: A Fast Track for

1511 Engineered Human Tissues in Drug Development. Cell Stem Cell 22, 310-324,

1512 doi:https://doi.org/10.1016/j.stem.2018.02.011 (2018).

1513163 McAleer, C. W. et al. Multi-organ system for the evaluation of efficacy and off-target

1514 toxicity of anticancer therapeutics. Science Translational Medicine 11, eaav1386,

1515 doi:10.1126/scitranslmed.aav1386 (2019).

1516164 Sances, S. et al. Human iPSC-Derived Endothelial Cells and Microengineered Organ-Chip

1517 Enhance Neuronal Development. Stem Cell Reports 10, 1222-1236,

1518 doi:https://doi.org/10.1016/j.stemcr.2018.02.012 (2018).

1519

1520 Increased calcium transients and mature gene expression seen in spinal motorneurons

1522165 Mulholland, T. et al. Drug screening of biopsy-derived spheroids using a self-generated

1523 microfluidic concentration gradient. Scientific Reports 8, 14672, doi:10.1038/s41598-

1524 018-33055-0 (2018).

1525166 Schutgens, F. et al. Tubuloids derived from human adult kidney and urine for personalized disease modeling. Nature Biotechnology 37, 303-313, doi:10.1038/s41587019-0048-8 (2019). 


\section{Contributions}

1530 LAL wrote and edited the manuscript and created the figures; CM, BB, DAT and CA reviewed 1531 and edited the manuscript.

1533 Competing Interests

1534 There are no competing interests.

1535

1536 Table of contents blurb

1537 Organs-on-chips (OoCs) could be useful at various stages of drug discovery and development; 1538 providing insight regarding human organ physiology in both normal and disease contexts, as 1539 well as accurately predicting developmental drug safety and efficacy. This Review discusses the 1540 advances that have enabled OoCs to demonstrate physiological relevance, and the challenges 1541 and opportunities that need to be tackled to tap the full potential of OoC utility for translational 1542 research. 